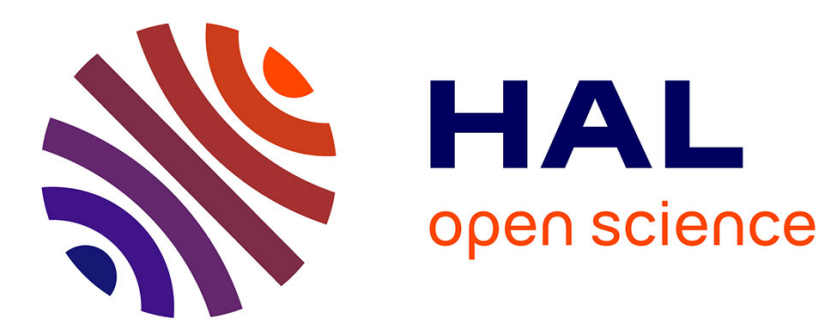

\title{
Robustness of stochastic discrete-time switched linear systems with application to control with shared resources
}

Luca Greco, Antoine Chaillet, Elena Panteley

\section{To cite this version:}

Luca Greco, Antoine Chaillet, Elena Panteley. Robustness of stochastic discrete-time switched linear systems with application to control with shared resources. IEEE Transactions on Automatic Control, 2015, 60 (12), pp.3168-3179. 10.1109/TAC.2015.2416921 . hal-00728093v2

\section{HAL Id: hal-00728093 \\ https://hal.science/hal-00728093v2}

Submitted on 30 Apr 2015

HAL is a multi-disciplinary open access archive for the deposit and dissemination of scientific research documents, whether they are published or not. The documents may come from teaching and research institutions in France or abroad, or from public or private research centers.
L'archive ouverte pluridisciplinaire $\mathbf{H A L}$, est destinée au dépôt et à la diffusion de documents scientifiques de niveau recherche, publiés ou non, émanant des établissements d'enseignement et de recherche français ou étrangers, des laboratoires publics ou privés.

$$
\text { Copyright }
$$




\title{
Robustness of stochastic discrete-time switched linear systems with application to control with shared resources
}

\author{
Luca Greco, Member, IEEE, Antoine Chaillet, Member, IEEE, and Elena Panteley
}

\begin{abstract}
Motivated by control applications relying on shared resources (such as computation time or bandwidth), we analyze the stability and robustness of discrete-time switched linear systems with stochastic commutations. We show that a wide class of shared resources control strategies can be modeled by a stochastic jump linear system involving two stochastic processes. The class of systems we study encompasses Markov chains and independent and identically distributed switching processes. For these systems, we recall existing definitions of stability and robustness, by relying on the input-to-state stability (ISS) property. We show that, for the class of systems under concern, $\delta$-moment stability is equivalent to $\delta$-moment ISS and that they both imply almost sure ISS. Several sufficient conditions are provided to guarantee these properties. Anytime control design for a Translational Oscillator/Rotational Actuator (TORA) system is used to illustrate all these concepts.
\end{abstract}

\section{INTRODUCTION}

The increasing demand of control functionalities from modern embedded systems (e.g. in the automotive domain [1]) calls for an increasingly heavy utilization of hardware resources. On the other hand, the need for keeping under control both hardware costs and system complexity clearly asks for an intense resource sharing. The same trend can be recognized in networked control systems (NCS), where economical concerns and ease of maintenance are forcing the adoption of a unique Ethernet-like network for the whole industrial plant, progressively eliminating specific subnets or fieldbuses [2]. The price to pay for resource sharing is a reduced predictability of the timing behavior: an application receives a different availability of resources and suffers timevarying delays depending on the interference suffered from other applications. This aspect becomes particularly apparent when the shared resource is the computation time provided by an embedded platform to many concurrent tasks. Traditional hard real-time approaches, based on worst case execution time (WCET) estimates, fall short of catching the large variability in computation time required by each task. Moreover, they suffer from an intrinsic conservativeness leading to poor performance, hardware underexploitation, and cost inefficiencies. A current trend in embedded system design is to relax hard schedulability constraints and introduce "softer" models of

This work has received funding from the European Union Seventh Framework Program [FP7/2007-2013] under grant agreement 257462 HYCON2 Network of excellence.

Authors are with: L2S - Supélec, 3 rue Joliot-Curie, 91192 Gif sur Yvette, France. E-mails: \{luca.greco, antoine.chaillet, elena.panteley\}@l2s.centralesupelec.fr. computation. Probabilistic modeling of real-time systems is by now a widely accepted approach to avoid overconservatism of deterministic (WCET-based) models, and it is the subject of an ample and growing literature [3], [4], [5]. Within stochastic models of Real-Time systems, the use of Markov chains (MC) (cf. e.g. [6], [7], [8]) is one of the most promising avenues to accurately compute the response time distribution and deadline miss probabilities of different tasks. This direction is also followed by a series of papers [9], [7], [10] of Lemmon and co-workers, where they consider performance of NCSs and embedded systems in a Firm Real-Time systems framework and introduce a stochastic model to describe the task dropout process based on MCs.

All the previous models belong to the class of Stochastic Jump Systems (SJS), namely switching systems whose commutations are ruled by a stochastic process. Commonly adopted stochastic processes are MCs and independent and identically distributed (i.i.d.) processes. Recently the need for a more general model has also emerged. In many practical cases (cf. e.g. [11], [12], [13], [14], [15], [16]), the evolution of a system involving shared resources cannot be adequately described in terms of a unique stochastic source. For instance, let us consider a system where different dynamics switch according to which (control) task is executed on a computational platform. The commutations among the different dynamics are induced by two distinct stochastic sources: the available computation time and the execution time of each task. Whence the need for a model accounting for the two stochastic sources.

As customary in control domain, finding conditions for ensuring the stability is the first concern for SJSs. There exists a wide gamut of stability definitions for stochastic systems (see for instance [17], [18], [19], [20]), whose precise comparison is usually a problematic task. Since our interest is mainly devoted to stochastic jump linear systems (SJLS) we will refer to the widely adopted stability definitions in [21], [22], [23], [24], [25], [26], [27]. However, these definitions do not explicitly take into account a prominent problem in control: ensuring robustness of the stability property with respect to perturbations or model inaccuracies. Input-to-state stability (ISS [28]) is a powerful tool to that aim. Traditional extensions of the deterministic ISS approach to stochastic systems mainly focus on continuous-time systems with a stochastic input (see for instance [29], [30], [31], [32]). Recently an important first step towards stochastic ISS for continuous-time SJS with stochastic commutations has appeared in [33], even if the general nonlinear framework there considered prevents from 
obtaining tight results for linear systems. A related robustness problem is addressed in [34], where a state feedback control is designed to ensure the almost sure stability of a continuoustime Markov jump linear system (MJLS) affected by structured uncertainties.

The goal of the present paper is twofold. First, we provide a formal description of a model of SJLS encompassing as special cases switching systems whose commutations are governed by MCs and i.i.d. processes. We enlighten the tight relation bonding the stochastic process adopted in the presented SJLS and the well known Hidden Markov Models (HMMs). We briefly present some concrete cases, where such model turns out essential to catch the complex and varying behavior of shared resource availability. Second, we make a connection between deterministic ISS results and the stochastic stability definitions in [21], [22], [23], [24], [25], [26], [27]. In particular we provide stochastic ISS definitions and easily testable conditions for the aforementioned more general model of discrete-time SJLSs. These definitions and conditions enjoy the nice property to coincide with the classical definitions and conditions in [21], [22], [23], [24], [25], [26], [27], when they are specialized to SJLSs governed by MCs or i.i.d. processes in the absence of disturbances. We finally show the effectiveness of our robust stability conditions on an interesting SJLS taken from anytime control literature.

We stress that, in this paper, we focus on discrete-time SJLSs with deterministic disturbances and stochastic commutations, while some classical MJLS models (see for instance [35], [19], [29], [30], [32]) consider both stochastic inputs and a stochastic source of commutations.

Notation: A function $\gamma: \mathbb{R}_{\geq 0} \rightarrow \mathbb{R}_{>0}$ is said to be of class $\mathcal{K}$ if it is continuous, zero at zero and strictly increasing. It is said of class $\mathcal{K}_{\infty}$ if it is also unbounded. A function $\beta: \mathbb{R}_{\geq 0} \times \mathbb{R}_{\geq 0} \rightarrow \mathbb{R}_{\geq 0}$ is said to be of class $\mathcal{K} \mathcal{L}$ if, given any $t \geq 0, \beta(\cdot, t) \in \mathcal{K}$ and, given any $s \geq 0, \beta(s, \cdot)$ is continuous, non-increasing and tends to zero as its argument tends to infinity. Given a symmetric positive definite matrix $M \in \mathbb{R}^{n \times n}$ we denote with $\lambda_{\min }(M)$ and $\lambda_{\max }(M)$ the smallest and the largest eigenvalue of $M$ respectively. Throughout the paper, $\|\cdot\|$ denotes both the Euclidean norm and the induced matrix norm: for $x \in \mathbb{R}^{n},\|x\|=\sqrt{x_{1}^{2}+\ldots+x_{n}^{2}}$ and, for $A \in \mathbb{R}^{n \times m},\|A\|=\max _{\|x\|=1}\|A x\|$. Given a locally bounded signal $d: \mathbb{Z}_{\geq 0} \rightarrow \mathbb{R}^{n}$, we define $\|d\|_{\infty}:=\sup _{k \in \mathbb{Z}_{\geq 0}}\|d(k)\|$.

\section{BASIC DEFINITIONS AND MotiVATIONS}

\section{A. System studied in this paper}

In this note we focus on the following stochastic jump linear system (SJLS)

$$
x(k+1)=A_{\varphi(k)} x(k)+B(x(k), k) d(k),
$$

where $k \in \mathbb{Z}_{\geq 0}, x(0)=x_{0} \in \mathbb{R}^{n}, A_{i} \in \mathbb{R}^{n \times n}$ for each $i \in J_{\varphi} \triangleq\{1, \ldots, N\}, N \in \mathbb{Z}_{>0}$ and $d: \mathbb{Z}_{\geq 0} \rightarrow \mathbb{R}^{p}$ is a deterministic, unknown but locally bounded disturbance. We assume that $B(x(k), k) \in \mathbb{R}^{n \times p}$ is a time-varying vector field for which ${ }^{1}$ there exists $\bar{b}>0$ such that

$$
\|B(x(k), k)\| \leq \bar{b}, \quad \forall k \in \mathbb{Z}_{\geq 0}, \forall x \in \mathbb{R}^{n} .
$$

The stochastic process $\{\varphi(\cdot)\}$, governing the index switching, takes values in the set $J_{\varphi}$ and is defined on the probability space $\left(\Omega_{\varphi}, \mathcal{F}_{\varphi}, \mathrm{P}_{\varphi}\right)$ where $\Omega_{\varphi}$ is the space of elementary events, $\mathcal{F}_{\varphi}$ is the associated sigma-algebra and $\mathrm{P}_{\varphi}$ is the probability measure. In order to fully describe the process $\{\varphi(\cdot)\}$ and motivated by several applications that we describe below (see Section II-B), let us introduce a discretetime finite-state time-homogeneous ${ }^{2}$ Markov Chain (FSHMC) $\{\sigma(\cdot)\}$ taking values in the set $J_{\sigma} \triangleq\{1, \ldots, M\}$, and defined on the probability space $\left(\Omega_{\sigma}, \mathcal{F}_{\sigma}, \mathrm{P}_{\sigma}\right)$. Due to the finite cardinality of the sets $J_{\sigma}$ and $J_{\varphi}$, the probability distributions associated to the processes $\{\varphi(\cdot)\}$ and $\{\sigma(\cdot)\}$ can be compactly represented at each step $k \in \mathbb{Z}_{\geq 0}$ by the row vectors $\pi_{\sigma}(k) \in S^{M-1}$ and $\pi_{\varphi}(k) \in S^{N-1}$, where $S^{N-1} \triangleq\left\{s=\left(s_{1}, \ldots, s_{N}\right) \in[0,1]^{N} \mid \sum_{i=1}^{N} s_{i}=1\right\}$ is the $(N-1)$-dimensional canonical stochastic simplex (and similarly for $M)$. The joint process $\{\sigma(\cdot), \varphi(\cdot)\}$, taking values in the set $J_{\sigma} \times J_{\varphi}$, is defined on the probability space $(\Omega, \mathcal{F}, \mathrm{P})$, where $\Omega \triangleq \Omega_{\sigma} \times \Omega_{\varphi}, \mathcal{F}$ is the sigma-algebra generated by the measurable rectangles $H_{\sigma} \times H_{\varphi}$ with $H_{\sigma} \in \mathcal{F}_{\sigma}$ and $H_{\varphi} \in \mathcal{F}_{\varphi}$ and $\mathrm{P}$ is a probability measure guaranteeing $\mathrm{P}\left\{H_{\sigma} \times H_{\varphi}\right\}=\mathrm{P}_{\sigma}\left\{H_{\sigma}\right\} \mathrm{P}_{\varphi}\left\{H_{\varphi}\right\}$ (see [36] page 231). Let us also denote with $\mathrm{E}\{\cdot\}$ the associated expectation operator.

By the Markov property we have that $\mathrm{P}\{\sigma(k+1)$ | $\sigma(k), \ldots, \sigma(0)\}=\mathrm{P}\{\sigma(k+1) \mid \sigma(k)\}$, hence a complete description of the FSH-MC $\{\sigma(\cdot)\}$ is given in terms of its transition probability matrix $P=\left(p_{l i}\right)_{l, i=1, \ldots, M}$, with $p_{l i} \triangleq \mathrm{P}\{\sigma(k+1)=i \mid \sigma(k)=l\}$, and its initial probability distribution $\pi_{\sigma}(0)=\pi_{\sigma 0}$. We consider a process $\{\varphi(\cdot)\}$ whose probability distribution $\pi_{\varphi} \in S^{N-1}$ is linearly related to the probability distribution $\pi_{\sigma} \in S^{M-1}$ of $\{\sigma(\cdot)\}$ by means of a row stochastic matrix $L=\left(\ell_{l j}\right)_{l=1, \ldots, M}$, with $\ell_{l j} \triangleq \mathrm{P}\{\varphi(k)=j \mid \sigma(k)=l\}$. Actually, we are assuming that the distribution of the process $\{\varphi(\cdot)\}$ at any step $k \in \mathbb{Z}_{\geq 0}$ depends only on $\sigma(k)$ and not on the previous values of $\{\sigma(\cdot)\}(\sigma(i), i<k)$ or $\{\varphi(\cdot)\}(\varphi(i), i<k)$. This means that $\mathrm{P}\{\varphi(k) \mid \varphi(k-1), \ldots, \varphi(0), \sigma(k), \ldots, \sigma(0)\}=$ $\mathrm{P}\{\varphi(k) \mid \sigma(k)\}$. Therefore, the probability distribution of the joint process $\{\sigma(\cdot), \varphi(\cdot)\}$ evolves according to the following dynamics

$$
\begin{aligned}
\pi_{\sigma}(k+1) & =\pi_{\sigma}(k) P \\
\pi_{\varphi}(k) & =\pi_{\sigma}(k) L .
\end{aligned}
$$

The equations (3)-(4) can be regarded as the description of a discrete-time LTI system without control, where $\pi_{\sigma}$ is the state vector and $\pi_{\varphi}$ is the output. The present stochastic model encompasses as special cases both FSH-MCs (if $J_{\varphi}=J_{\sigma}$ and

\footnotetext{
${ }^{1}$ An interesting case is obtained when the input matrix switches in the same way as the $A$ matrix, namely for $B(x(k), k)=B_{\varphi(k)}$, in which case $\bar{b}=\max _{i \in J_{\varphi}}\left\|B_{i}\right\|$.

${ }^{2}$ Time-homogeneous Markov Chains (sometimes referred to as stationary Markov Chains) are characterized by transition probabilities which are independent of time.
} 
$L$ is chosen as the identity matrix) and, in particular, independent and identically distributed (i.i.d.) processes. It is worth noting that the model (3)-(4) can be readily interpreted as a Hidden Markov Model (HMM) [37], where the (hidden) state of the $\mathrm{MC}\{\sigma(\cdot)\}$ is not directly observable and the observation process $\{\varphi(\cdot)\}$ is used to reconstruct such state. We stress, however, that our interest here is not about the estimation and reconstruction problems, which are already widely studied in literature (see for instance [38], [39], [40]), but rather in robust stability problems arising when the commutations of a switching system are ruled by a HMM.

If the FSH-MC $\{\sigma(\cdot)\}$ is also irreducible and aperiodic (FSHIA-MC) (see [41, Section 8.4] and [36, Section 8]), then there exists a unique invariant probability distribution (i.p.d.) $\bar{\pi}_{\sigma}$ such that $\lim _{k \rightarrow \infty} \pi_{\sigma}(k)=\bar{\pi}_{\sigma}$ for any $\pi_{\sigma}(0)$. Namely, $\{\sigma(\cdot)\}$ is an ergodic process with a unique ergodic class (see [36, Section 24] and [41, Section 8.4]). In this case the process $\{\varphi(\cdot)\}$ inherits the ergodic property of $\{\sigma(\cdot)\}$ and a unique i.p.d. $\bar{\pi}_{\varphi} \triangleq \bar{\pi}_{\sigma} L$ exists for that process.

\section{B. Motivating examples}

The stochastic model (3)-(4) describes a wide range of different systems. It is especially relevant in modeling a limited resource shared among competing users. The MC $\{\sigma(\cdot)\}$ can represent the quantized amount of resource, in the finite set $J_{\sigma}$, available at each instant $k \in \mathbb{Z}_{\geq 0}$, while the process $\{\varphi(\cdot)\}$ can represent the index of the user using the resource at that instant. With such an interpretation $\pi_{\varphi}(k)$ is the probability of each user to exclusively use the resource at time $k$ and the $(i, j)$-th entry of $L$ represents the probability of the $j$-th user to need an amount of resource equal to the $i$-th value in the set $J_{\sigma}$. We further illustrate this in practical examples in control with shared resources.

Example 1. The following system, considered in [12], fits the previous description. In this system, the shared resource is the computation time offered by a multitasking embedded unit for the execution of a periodic control task. In order to cope with the fluctuations in the available computation time, the control task is designed according to the anytime paradigm. Namely, it is decomposed in a finite set of ordered subroutines $\Gamma_{j}, j \in J_{\varphi}$, guaranteeing, as $j$ increases, an increasing performance but requiring an increasing computation time. Due to the lack of deterministic information on the computation time availability in each period, the subroutines are forced to execute sequentially, i.e. the computation of $\Gamma_{j}$ cannot start until the computation of $\Gamma_{j-1}$ has terminated. For sake of clarity, let us focus on a particular implementation of the anytime control paradigm involving a special modular structure (see Figure 1 for the case of two subroutines). A detailed case study is provided in section IV.

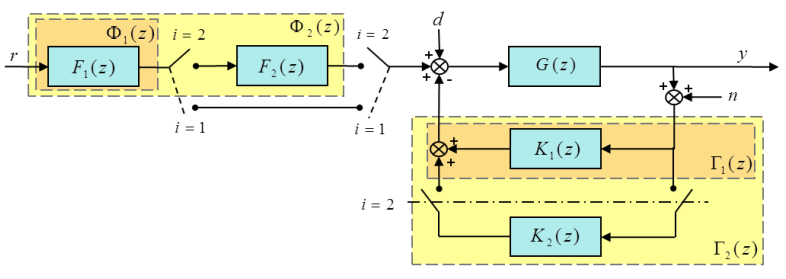

Fig. 1. Schematic representation of the system and the anytime control. When the switches are in the $i=1(i=2)$ position the controller $\Gamma_{1}\left(\Gamma_{2}\right)$ and the prefilter $\Phi_{1}\left(\Phi_{2}\right)$ are active.

In the present implementation the subroutine $\Gamma_{1}$ coincides with the controller $K_{1}$, while the subroutine $\Gamma_{j}$ is the sum of all component controllers from $K_{1}$ to $K_{j}$. In order to improve the overall performance, some composite prefilters can be added, that is $\Phi_{1}=F_{1}$ and, in general, $\Phi_{j}=F_{1}+\cdots+F_{j}$. The computation starts with $K_{1}$ and $F_{1}$ and proceeds by progressively adding modules according to the available computation time. In case sufficient computation time is available, the controller $\Gamma_{j}$ and the prefilter $\Phi_{j}$ are executed, otherwise, in case of premature interruption, an intermediate controller and filter are executed (see [12, Sec. VII] for further information on anytime control implementation and tracking problems). The ensuing closed-loop system is described by the SJLS (1), (3)-(4). In this setting the FSHIA-MC $\{\sigma(\cdot)\}$ describes the amount of available computation time and the process $\{\varphi(\cdot)\}$ the highest index of a schedulable controller, i.e. the subroutine and prefilter whose execution time is the largest among those shorter than the available time. The execution time $T^{j}$ required to compute sequentially all component controllers from $K_{1}$ to $K_{j}$ and all component prefilters from $F_{1}$ to $F_{j}$ is modeled as a discrete time i.i.d. process taking values in the set $J_{\sigma}$. If we represent the stationary probability distribution of $T^{j}$ with the row vector $\pi_{T^{j}}=\left[\pi_{T_{1}^{j}}, \cdots, \pi_{T_{M}^{j}}\right]$ and the associated cumulative probability distribution with $\mu_{T^{j}}=\left[\pi_{T_{1}^{j}}, \pi_{T_{1}^{j}}+\pi_{T_{2}^{j}}, \cdots, \sum_{h=1}^{M} \pi_{T_{h}^{j}}\right]$, we can derive the matrix $L$ in (4) as follows (see [12])

$$
L=\left[\begin{array}{c}
\mu_{T^{1}}-\mu_{T^{2}} \\
\vdots \\
\mu_{T^{M-1}}-\mu_{T^{M}} \\
\mu_{T^{M}}
\end{array}\right]^{T} .
$$

Thus the $(i, j)$-th entry of $L$ represents the probability of executing all subroutines and prefilters until the $j$-th ones if an amount $i$ of computation time is available. As a consequence if $\varphi(k)=j$ then in the $k$-th period all controllers $\Gamma_{l}$ and prefilters $\Phi_{l}, l \leq j$, but no controller $\Gamma_{h}$ and prefilter $\Phi_{h}$, $h>j$, can be executed.

The model (3)-(4) is open to a dual interpretation. Still in the case of a shared resource among different users, the MC $\{\sigma(\cdot)\}$ can represent the index of the user exploiting the resource at any $k \in \mathbb{Z}_{\geq 0}$, while the process $\{\varphi(\cdot)\}$ may represent the amount of resource used at time $k$.

Example 2. In Section IV of [13] this model is used to describe the overall computation time used by a set of concurrent periodic tasks. For the sake of simplicity, let us focus on a single task only. Such a task is assumed to have a finite 
number of working modes, which, in this description, play the role of the users. In each period $k$ the task can be in one of $M$ possible modes defined by the set $J_{\sigma}$. The change in time of the modes is assumed to be ruled by a FSHIA-MC $\{\sigma(\cdot)\}$. According to each mode, the task can require a certain amount of computation time assuming values in the finite set $J_{\varphi}$ and described by an i.i.d. process. In this case, the $(i, j)$-th entry of $L$ represents the probability of the task to require an amount $j$ of computation time during the mode $i$. In the cited paper this model is used to provide a stochastic description of the computation time left available by higher priority tasks to the execution of a lower priority control task.

A similar model is presented in Section VII of [16], where a FSHIA-MC rules the transition among processor states (or modes) and a stochastic process (analogous to $\{\varphi(\cdot)\}$ here) describes the length of the control sequence computed at each step.

It is interesting to note that the presented model bears some similarities also with the one presented in [42]. In the latter paper a system experiences deterministic switchings among different working modes. Each mode is associated with a different MJLS with distinct dynamic matrices and stochastic properties. The ensuing switching MJLS is ensured to be mean square stable provided that some dwell-time conditions hold. Instead, we consider here a unique set of dynamic matrices. At each step, the choice of the applied matrix in this set is ruled by a given stochastic process. This stochastic process depends on the present mode, and the evolution from one mode to the other is itself ruled by another stochastic process.

We remark that the model (3)-(4) is flexible enough to also encompass embedded control systems affected by variable computation delays and networked systems.

Example 3. In [14], [15] a class of scheduling algorithms, known as adaptive reservations, is applied to control problems. Adaptive reservations allow the formulation of a discrete time dynamic model for the evolution of the delay potentially experienced by a control task. If the computation time of the task is an i.i.d. process and the bandwidth allocation policy is a Markov process, then the delay is described by a MC $\{\sigma(\cdot)\}$. According to the delay, namely to the state of the MC $\{\sigma(\cdot)\}$, a scheduling policy decides whether executing the delayed control task or dropping the computation. At each time step the system can be either in open loop (drop case) or in closed loop with a specific delay. Then, if a finite discrete set of values is considered for the delay, the switching among the different systems is ruled by a stochastic process $\{\varphi(\cdot)\}$, which encodes the outcome of the scheduling policy.

Example 4. In [11] a simple model of network delay dynamics is presented. In such a model a three-state MC is used to describe different network load conditions (modes): low, medium and high. Associated to each mode there is an i.i.d. process describing the delay probability for different values of delays. Clearly larger delays are more likely to occur in high than in low load conditions. If we consider a discrete and finite set of possible delays, we can easily fit the model in [11] with the one here presented. It suffices to identify the MC with $\{\sigma(\cdot)\}$ and to stack the three delay distributions (considered as vectors) to build the $L$ matrix. The ensuing process $\{\varphi(\cdot)\}$ will then represent at each step $k$ the transmission delay experienced by a measurement or a control packet.

Many other works modeling the network traffic fit, or can be adapted to fit, the present stochastic framework. Here we concisely refer to some of them. In [?] a HMM is proposed to model the packet loss process in TCP channels. The MC describes the evolution of the network state, while the block/pass event is determined by different binary probability distributions associated with each state. The paper [?] aims at modeling the IP traffic exhibiting long-range dependence. There, a discrete-time batch Markov arrival process is used to jointly characterize the packet arrival process and the packet size distribution. In particular, a discrete-time Markov modulated Poisson process (dMMPP) accounts for the packet arrivals and a probability distribution associated to the MC state of the dMMPP accounts for the packet size. In [43], the authors employ a HMM to describe the joint delay and loss dynamics in periodic UDP traffic. A HMM is used also in [?] to provide a characterization of Internet traffic at a packet level, namely to jointly describe the Inter Packet Time and the Packet Size processes.

\section{Stability and robustness definitions}

In Definition 5 we adapt classical stochastic stability definitions, such as those in [21], [22], [23], [24], to fit the SJLS system (1) governed by the stochastic process $\{\sigma(\cdot), \varphi(\cdot)\}$. We denote with $x(k, d)$ the stochastic process solution $x\left(k, x_{0}, d, \omega\right), \omega \in \Omega$ of the difference equation (1) with non random initial condition $x(0)=x_{0} \in \mathbb{R}^{n}$ and input signal $d: \mathbb{Z}_{\geq 0} \rightarrow \mathbb{R}^{p}$. when the case is explicit enough, we might just write $x(k)$ to denote $x(k, d)$.

Definition 5. Given a constant $\delta \in \mathbb{R}_{>0}$ and the set of admissible initial distributions $\Phi \triangleq\left\{\left(\pi_{\sigma 0}, \pi_{\varphi 0}\right) \in S^{N+M-2} \mid \pi_{\varphi 0}=\pi_{\sigma 0} L, \pi_{\sigma 0} \in S^{M-1}\right\}$, the system (1) with $d=0$ and driven by the stochastic process $\{\sigma(\cdot), \varphi(\cdot)\}$ with dynamics (3)-(4) is said to be:

1) $\delta$-moment globally asymptotically stable $(\delta-G A S)$ if, for any $x_{0} \in \mathbb{R}^{n}$ and any initial distribution $\left(\pi_{\sigma 0}, \pi_{\varphi 0}\right) \in \Phi$ of $\{\sigma(\cdot), \varphi(\cdot)\}$,

$$
\lim _{k \rightarrow \infty} \mathrm{E}\left\{\|x(k, 0)\|^{\delta}\right\}=0 ;
$$

2) $\delta$-moment globally exponentially stable $(\delta$-GES) if there exist constants $a, b \in \mathbb{R}_{>0}$ such that for any $x_{0} \in \mathbb{R}^{n}$ and any initial distribution $\left(\pi_{\sigma 0}, \pi_{\varphi 0}\right) \in \Phi$ of $\{\sigma(\cdot), \varphi(\cdot)\}$

$$
\mathrm{E}\left\{\|x(k, 0)\|^{\delta}\right\} \leq a\left\|x_{0}\right\|^{\delta} \mathrm{e}^{-b k}, \quad \forall k \in \mathbb{Z}_{\geq 0} ;
$$

3) almost surely (with probability one) globally stable (as$G S)$ if for any $x_{0} \in \mathbb{R}^{n}$ and any initial distribution $\left(\pi_{\sigma 0}, \pi_{\varphi 0}\right) \in \Phi$ of $\{\sigma(\cdot), \varphi(\cdot)\}$

$$
\mathrm{P}\left\{\lim _{k \rightarrow \infty}\|x(k, 0)\|=0\right\}=1 .
$$

We stress that, despite their names, $\delta$-GAS and as-GS actually address the convergence of solutions of the system 
(1) to the origin, rather than stability issues. Nonetheless, for stochastic linear systems $\delta$-GAS and $\delta$-GES are equivalent properties (see for instance [25], [26], [27], [23]). Hence the convergence property in Definition 5.1 actually implies the stability contained in Definition 5.2. In order to analyze the robustness of system (1), we introduce the following new stability definitions.

Definition 6. Given a constant $\delta \in \mathbb{R}_{>0}$ and the set $\Phi$ of admissible initial distributions as in Definition 5 , the system (1) driven by the stochastic process $\{\sigma(\cdot), \varphi(\cdot)\}$ with dynamics (3)-(4) is said to be:

1) $\delta$-moment input-to-state stable $(\delta$-ISS) if there exist functions $\beta \in \mathcal{K} \mathcal{L}$ and $\gamma \in \mathcal{K}_{\infty}$ such that for any $x_{0} \in \mathbb{R}^{n}$, any initial distribution $\left(\pi_{\sigma 0}, \pi_{\varphi 0}\right) \in \Phi$ of $\{\sigma(\cdot), \varphi(\cdot)\}$ and any locally bounded $d: \mathbb{Z}_{\geq 0} \rightarrow \mathbb{R}^{p}$

$$
\mathrm{E}\left\{\|x(k, d)\|^{\delta}\right\} \leq \beta\left(\left\|x_{0}\right\|, k\right)+\gamma\left(\|d\|_{\infty}\right), \quad \forall k \in \mathbb{Z}_{\geq 0} ;
$$

2) $\delta$-moment exponentially input-to-state stable $(\delta$-EISS) if there exist constants $a, b \in \mathbb{R}_{>0}$ and a function $\gamma \in$ $\mathcal{K}_{\infty}$ such that for any $x_{0} \in \mathbb{R}^{n}$, any initial distribution $\left(\pi_{\sigma 0}, \pi_{\varphi 0}\right) \in \Phi$ of $\{\sigma(\cdot), \varphi(\cdot)\}$ and any locally bounded $d: \mathbb{Z}_{\geq 0} \rightarrow \mathbb{R}^{p}$

$\mathrm{E}\left\{\|x(k, d)\|^{\delta}\right\} \leq a\left\|x_{0}\right\|^{\delta} \mathrm{e}^{-b k}+\gamma\left(\|d\|_{\infty}\right), \quad \forall k \in \mathbb{Z}_{\geq 0} ;$

3) almost surely (with probability one) input-to-state stable (as-ISS) if there exist functions $\beta \in \mathcal{K} \mathcal{L}$ and $\gamma \in \mathcal{K}_{\infty}$ such that for any $x_{0} \in \mathbb{R}^{n}$, any initial distribution $\left(\pi_{\sigma 0}, \pi_{\varphi 0}\right) \in \Phi$ of $\{\sigma(\cdot), \varphi(\cdot)\}$ and any locally bounded $d: \mathbb{Z}_{\geq 0} \rightarrow \mathbb{R}^{p}$

$\mathrm{P}\left\{\|x(k, d)\| \leq \beta\left(\left\|x_{0}\right\|, k\right)+\gamma\left(\|d\|_{\infty}\right)\right\}=1, \quad \forall k \in \mathbb{Z}_{\geq 0}$.

As pointed out in [26], the Definitions 5 are stronger than the weak stability definitions in [17], as they require to be independent of the initial distribution. Definitions 6 inherit the same property. It is also worth noting that the above $\delta$ ISS definition is close to the ISS in $L_{1}$ estimate at switching instants introduced for continuous time switching systems in [33].

The results derived in this paper aim at providing some robust stability guarantees for the SJLS (1), (3)-(4). They can be used to ensure that the presence of disturbances do not disrupt the stability properties enjoyed by the considered systems. Once established that the system of interest fits the presented model, the tools can be used to verify the robust stability property for a given distribution of the shared resource. It is worth noting that for "given distribution" we mean a distribution whose stochastic characterization is known. There is no need for the knowledge of any specific realization (i.e. sampling path) of the stochastic process.

Remark 7. Due to the special structure of $\Phi$, which imposes a linear relation between $\pi_{\varphi 0}$ and $\pi_{\sigma 0}$, in the following we will slightly abuse the notation and refer to the probability measure $\mathrm{P}_{\left(\pi_{\sigma 0}, \pi_{\varphi 0}\right)}\{\cdot\}$, induced by any initial distribution $\left(\pi_{\sigma 0}, \pi_{\varphi 0}\right) \in$ $\Phi$, as $\mathrm{P}_{\pi_{\sigma 0}}\{\cdot\}$.

\section{MAIN RESUltS}

On the one hand, the systems evoked in Section II-B demonstrate the need to develop analytical tools guaranteeing stability and robustness of SJLS of the form (1). On the other hand, Section II-C underlines the diversity of existing notions of stability for such systems. This section therefore aims at exhibiting the tight links existing between the stability and robustness notions recalled above for the specific class of systems (1), (3)-(4), and at developing practical conditions to establish them.

\section{A. $\delta$-ISS and 2-ISS}

Asymptotic second moment stability (2-GAS) and exponential second moment stability (2-GES), for systems without input and driven by a Markov chain, have been shown to be equivalent and to imply the almost sure stability (as-GS) ([25], [26], [27]). Similar properties hold for $\delta$-GAS and $\delta$ GES (see for instance [44], [23]). In this section we prove similar equivalences for the more general class of systems (1), (3)-(4).

Theorem 8. For the system (1) driven by the stochastic process $\{\sigma(\cdot), \varphi(\cdot)\}$, whose distributions are described by the evolutions (3) and (4), $\delta$-GAS, $\delta$-GES, $\delta$-ISS and $\delta$-EISS are equivalent.

Proof: We will prove the following chain of implications: $\delta$-GAS $\Rightarrow \delta$-GES $\Rightarrow \delta$-EISS $\Rightarrow \delta$-ISS $\Rightarrow \delta$-GAS. The latter two implications are easily verified, thus we need to prove only the first two: $\delta$-GAS $\Rightarrow \delta$-GES $\Rightarrow \delta$-EISS.

\section{$\delta$-GAS $\Rightarrow \delta$-GES part.}

This part of the proof is close to the proof of Theorem 4.1 of [23]. For any $k \in \mathbb{Z}_{\geq 0}$, any $x_{0} \in \mathbb{R}^{n}$ and $d \equiv 0$ the evolution of the system (1) is given by $x(k, 0)=A_{\varphi(k-1)} \cdots A_{\varphi(0)} x_{0}$. Hence, for any initial distribution $\pi_{\sigma 0} \in S^{M-1}$ and the corresponding distribution $\pi_{\varphi 0}=\pi_{\sigma 0} L$, we have

$$
\mathrm{E}_{\pi_{\sigma 0}}\left\{\|x(k, 0)\|^{\delta}\right\} \leq \mathrm{E}_{\pi_{\sigma 0}}\left\{\left\|A_{\varphi(k-1)} \cdots A_{\varphi(0)}\right\|^{\delta}\right\}\left\|x_{0}\right\|^{\delta}
$$

with $\mathrm{E}_{\pi_{\sigma 0}}\{\cdot\}$ denoting the expectation value with respect to the probability measure $\mathrm{P}_{\pi_{\sigma 0}}\{\cdot\}$ (see Remark 7). By the definition of expectation and letting $\pi_{\sigma 0} \triangleq\left(\pi_{\sigma 0,1}, \ldots, \pi_{\sigma 0, M}\right)$, we have

$$
\begin{array}{r}
\mathrm{E}_{\pi_{\sigma 0}}\left\{\left\|A_{\varphi(k-1)} \cdots A_{\varphi(0)}\right\|^{\delta}\right\}= \\
\sum_{i_{0}=1}^{M} \pi_{\sigma 0, i_{0}} \mathrm{E}_{e_{i_{0}}}\left\{\left\|A_{\varphi(k-1)} \cdots A_{\varphi(0)}\right\|^{\delta}\right\},
\end{array}
$$

where $e_{i_{0}}$ is the $i_{0}$-th vector of the canonical base of $\mathbb{R}^{M}$. We will show that the quantity $\mathrm{E}_{e_{i_{0}}}\left\{\left\|A_{\varphi(k-1)} \cdots A_{\varphi(0)}\right\|^{\delta}\right\}$ is independent of the initial distribution and depends only on the arbitrary index $i_{0}$. For ease of notation, in what follows we will write $\varphi=j_{k-1}, j_{0}$ and $\sigma=i_{k-1}, i_{0}$ instead of $\varphi(k-1)=$ $j_{k-1}, \ldots, \varphi(0)=j_{0}$ and $\sigma(k-1)=i_{k-1}, \ldots, \sigma(0)=i_{0}$ 
respectively. We have

$$
\begin{aligned}
& \mathrm{E}_{e_{i_{0}}}\left\{\left\|A_{\varphi(k-1)} \cdots A_{\varphi(0)}\right\|^{\delta}\right\} \\
& =\sum_{j_{0}, \ldots, j_{k-1}}\left\|A_{j_{k-1}} \cdots A_{j_{0}}\right\|^{\delta} \mathrm{P}\left\{\varphi=j_{k-1}, j_{0} \mid \sigma(0)=i_{0}\right\} \\
& =\sum_{j_{0}, \ldots, j_{k-1}}\left\|A_{j_{k-1}} \cdots A_{j_{0}}\right\|^{\delta} \\
& \times \sum_{i_{1}, \ldots, i_{k-1}} \mathrm{P}\left\{\varphi=j_{k-1}, j_{0} \mid \sigma=i_{k-1}, i_{0}\right\} \\
& \times \mathrm{P}\left\{\sigma=i_{k-1}, i_{1} \mid \sigma(0)=i_{0}\right\} .
\end{aligned}
$$

Moreover

$$
\begin{aligned}
& \mathrm{P}\left\{\varphi=j_{k-1}, j_{0} \mid \sigma=i_{k-1}, i_{0}\right\} \\
& =\mathrm{P}\left\{\varphi(k-1)=j_{k-1} \mid \varphi=j_{k-2}, j_{0}, \sigma=i_{k-1}, i_{0}\right\} \\
& \times \mathrm{P}\left\{\varphi=j_{k-2}, j_{0} \mid \sigma=i_{k-1}, i_{0}\right\} \\
& =\mathrm{P}\left\{\varphi(k-1)=j_{k-1} \mid \sigma(k-1)=i_{k-1}\right\} \\
& \times \mathrm{P}\left\{\varphi=j_{k-2}, j_{0} \mid \sigma=i_{k-1}, i_{0}\right\},
\end{aligned}
$$

where we used the fact that $\varphi(k-1)$ is dependent of $\sigma(k-1)$ but independent of the previous values of $\{\varphi(\cdot)\}$ and $\{\sigma(\cdot)\}$. By iterating the previous relation and recalling that $\ell_{l j} \triangleq \mathrm{P}\{\varphi(k)=j \mid \sigma(k)=l\}$, we find

$$
\mathrm{P}\left\{\varphi=j_{k-1}, j_{0} \mid \sigma=i_{k-1}, i_{0}\right\}=\ell_{i_{0} j_{0}} \cdots \ell_{i_{k-1} j_{k-1}} .
$$

Proceeding in a similar fashion we find also

$$
\mathrm{P}\left\{\sigma=i_{k-1}, i_{1} \mid \sigma(0)=i_{0}\right\}=p_{i_{0} i_{1}} \cdots p_{i_{k-2} i_{k-1}} .
$$

Plugging (7) and (8) in (6) yields

$$
\begin{aligned}
& \mathrm{E}_{e_{i_{0}}}\left\{\left\|A_{\varphi(k-1)} \cdots A_{\varphi(0)}\right\|^{\delta}\right\} \\
& =\sum_{i_{1}, \ldots, i_{k-1}} \sum_{j_{0}, \ldots, j_{k-1}} p_{i_{0} i_{1}} \cdots p_{i_{k-2} i_{k-1}} \times \ell_{i_{0} j_{0}} \cdots \ell_{i_{k-1} j_{k-1}} \\
& \times\left\|A_{j_{k-1}} \cdots A_{j_{0}}\right\|^{\delta}
\end{aligned}
$$

which is clearly independent of the initial distribution $\pi_{\sigma 0}$.

According to Definition 5-1, we have that

$$
\lim _{k \rightarrow \infty} \mathrm{E}_{\pi_{\sigma 0}}\left\{\left\|A_{\varphi(k-1)} \cdots A_{\varphi(0)}\right\|^{\delta}\right\}=0 .
$$

Recalling (5) and that $\mathrm{E}_{e_{i_{0}}}\left\{\left\|A_{\varphi(k-1)} \cdots A_{\varphi(0)}\right\|^{\delta}\right\}$ is independent of $\pi_{\sigma 0}$, the previous limit implies that $\lim _{k \rightarrow \infty} \mathrm{E}_{e_{i_{0}}}\left\{\left\|A_{\varphi(k-1)} \cdots A_{\varphi(0)}\right\|^{\delta}\right\}=0$ for every $e_{i_{0}}$. Hence, the following limit is also true

$$
\lim _{k \rightarrow \infty} \sum_{i=1}^{M} \mathrm{E}_{e_{i_{0}}}\left\{\left\|A_{\varphi(k-1)} \cdots A_{\varphi(0)}\right\|^{\delta}\right\}=0 .
$$

Such a limit is actually independent of the initial distribution and we can refer to it, with a slight abuse of notation, by means of the generic expectation operator

$$
\lim _{k \rightarrow \infty} \mathrm{E}\left\{\left\|A_{\varphi(k-1)} \cdots A_{\varphi(0)}\right\|^{\delta}\right\}=0 .
$$

We can argue by the compactness of $S^{M-1}$ that, for any $\varepsilon \in$ $(0,1)$, there exists $Q=Q(\varepsilon) \in \mathbb{Z}_{>0}$ such that

$$
\mathrm{E}\left\{\left\|A_{\varphi(q-1)} \cdots A_{\varphi(0)}\right\|^{\delta}\right\} \leq \varepsilon, \forall q \geq Q
$$

By the independence of the initial distribution and the timehomogeneity property of the composite process $\{\sigma(\cdot), \varphi(\cdot)\}$, the previous inequality holds true at any instant $s \in \mathbb{Z}_{>0}$. Hence, we have

$$
\mathrm{E}\left\{\left\|A_{\varphi(q+s-1)} \cdots A_{\varphi(s)}\right\|^{\delta}\right\} \leq \varepsilon, \forall s \geq 0, \forall q \geq Q .
$$

We can also define a constant $c>1$ such that we have $\mathrm{E}\left\{\left\|A_{\varphi(h+s-1)} \cdots A_{\varphi(s)}\right\|^{\delta}\right\} \leq c, \forall s \in \mathbb{Z}_{>0}, \forall h \in(0, Q) \cap \mathbb{Z}$

As in the proof of Theorem 4.1 of [23] and using again the time-homogeneity property of $\{\sigma(\cdot), \varphi(\cdot)\}$, we have for that the total expectation (5) can be sliced in $r$ pieces of length $Q$ and one piece of length $h$ for any $k=r Q+h, h, r \in \mathbb{Z}_{>0}$, $h<Q$ :

$$
\begin{aligned}
& \mathrm{E}_{\pi_{\sigma 0}}\left\{\left\|A_{\varphi(k-1)} \cdots A_{\varphi(0)}\right\|^{\delta}\right\} \leq \mathrm{E}\left\{\left\|A_{\varphi(k-1)} \cdots A_{\varphi(0)}\right\|^{\delta}\right\} \\
& =\sum_{i_{0}, \ldots, i_{Q-1}} \sum_{j_{0}, \ldots, j_{Q-1}} p_{i_{0} i_{1}} \cdots p_{i_{Q-2} i_{Q-1}} \\
& \times \ell_{i_{0} j_{0}} \cdots \ell_{i_{Q-1} j_{Q-1}}\left\|A_{j_{Q-1}} \cdots A_{j_{0}}\right\|^{\delta} \times \cdots \\
& \times \sum_{i_{(r-1) Q}, \ldots, i_{r Q-1}} \sum_{j_{(r-1) Q}, \ldots, j_{r Q-1}} p_{i_{(r-1) Q} i_{(r-1) Q+1}} \cdots p_{i_{r Q-2} i_{r Q-1}} \\
& \times \ell_{i_{(r-1) Q} j_{(r-1) Q}} \cdots \ell_{i_{r Q-1} j_{r Q-1}}\left\|A_{j_{r Q-1}} \cdots A_{j_{(r-1) Q}}\right\|^{\delta} \\
& \times \sum_{i_{r Q}, \ldots, i_{r Q+h} j_{r Q}, \ldots, j_{r Q+h}} p_{i_{r Q} i_{r Q+1}} \cdots p_{i_{r Q+h-1} i_{r Q+h}} \\
& \times \ell_{i_{r Q} j_{r Q}} \cdots \ell_{i_{r Q+h} j_{r Q+h}}\left\|A_{j_{r Q+h}} \cdots A_{j_{r Q}}\right\|^{\delta} .
\end{aligned}
$$

Therefore

$$
\begin{aligned}
& \mathrm{E}\left\{\left\|A_{\varphi(k-1)} \cdots A_{\varphi(0)}\right\|^{\delta}\right\} \leq \mathrm{E}\left\{\left\|A_{\varphi(Q-1)} \cdots A_{\varphi(0)}\right\|^{\delta}\right\} \times \cdots \\
& \times \mathrm{E}\left\{\left\|A_{\varphi(r Q-1)} \cdots A_{\varphi((r-1) Q)}\right\|^{\delta}\right\} \mathrm{E}\left\{\left\|A_{\varphi(r Q+h)} \cdots A_{\varphi(r Q)}\right\|^{\delta}\right\}
\end{aligned}
$$

and, exploiting (12) and (13):

$$
\mathrm{E}\left\{\left\|A_{\varphi(k-1)} \cdots A_{\varphi(0)}\right\|^{\delta}\right\} \leq c \varepsilon^{r}=a \mathrm{e}^{-b k},
$$

with $a \triangleq c \varepsilon^{-\frac{h}{Q}}$ and $b \triangleq-\frac{1}{Q} \log \varepsilon$. Finally, we have that $\mathrm{E}\left\{\|x(k, 0)\|^{\delta}\right\} \leq a\left\|x_{0}\right\|^{\delta} \mathrm{e}^{-b k}$, which establishes $\delta$-GES.

$\delta$-GES $\Rightarrow \delta$-EISS part.

For any $k \in \mathbb{Z}_{\geq 0}$ and any $x_{0} \in \mathbb{R}^{n}$ we can write

$$
\begin{aligned}
x(k, d) & =A_{\varphi(k-1)} \cdots A_{\varphi(0)} x_{0}+A_{\varphi(k-1)} \cdots A_{\varphi(1)} B\left(x_{0}, 0\right) d(0) \\
& +\cdots+A_{\varphi(k-1)} B(x(k-2), k-2) d(k-2) \\
& +B(x(k-1), k-1) d(k-1) .
\end{aligned}
$$

Notice that the constant $\bar{b}$ in (2) can be assumed greater than 1 without loss of generality. Therefore, we get

$$
\begin{aligned}
\|x(k, d)\|^{\delta} & \leq\left(\left\|A_{\varphi(k-1)} \cdots A_{\varphi(0)}\right\|\left\|x_{0}\right\|\right. \\
& +\bar{b}\|d\|_{\infty}\left(1+\left\|A_{\varphi(k-1)}\right\|+\cdots\right. \\
& \left.\left.+\left\|A_{\varphi(k-1)} \cdots A_{\varphi(1)}\right\|\right)\right)^{\delta} .
\end{aligned}
$$

Moreover, there exists a constant $c_{1} \in \mathbb{R}_{>0}$ such that

$$
\begin{aligned}
\|x(k, d)\|^{\delta} & \leq c_{1}\left\|A_{\varphi(k-1)} \cdots A_{\varphi(0)}\right\|^{\delta}\left\|x_{0}\right\|^{\delta} \\
& +c_{1} \bar{b}^{\delta}\|d\|_{\infty}^{\delta}\left(1+\left\|A_{\varphi(k-1)}\right\|+\cdots\right. \\
& \left.+\left\|A_{\varphi(k-1)} \cdots A_{\varphi(1)}\right\|\right)^{\delta} .
\end{aligned}
$$


For $\delta \leq 1$ we can choose $c_{1}=1$ in light of the subadditivity of the concave function $(\cdot)^{\delta}$. For $\delta>1$ the function $(\cdot)^{\delta}$ is convex and we can exploit such property to find $c_{1}=2^{\delta-1}$. Let us focus now on the second term in the previous bound and add the non-negative term $\left\|A_{\varphi(k-1)} \cdots A_{\varphi(0)}\right\|$ :

$$
\begin{aligned}
& \left(1+\left\|A_{\varphi(k-1)}\right\|+\left\|A_{\varphi(k-1)} A_{\varphi(k-2)}\right\|+\cdots\right. \\
& \left.+\left\|A_{\varphi(k-1)} \cdots A_{\varphi(1)}\right\|\right)^{\delta} \leq \\
& \left(1+\left\|A_{\varphi(k-1)}\right\|+\left\|A_{\varphi(k-1)} A_{\varphi(k-2)}\right\|+\cdots\right. \\
& \left.\left\|A_{\varphi(k-1)} \cdots A_{\varphi(1)}\right\|+\left\|A_{\varphi(k-1)} \cdots A_{\varphi(0)}\right\|\right)^{\delta} .
\end{aligned}
$$

We claim that there exists a constant $c_{2} \in \mathbb{R}_{>0}$ and coefficients $\beta_{i} \in \mathbb{R}_{>0}, i=1, \ldots, k+1$, such that

$$
\begin{aligned}
& \left(1+\left\|A_{\varphi(k-1)}\right\|+\left\|A_{\varphi(k-1)} A_{\varphi(k-2)}\right\|+\cdots\right. \\
& \left.+\left\|A_{\varphi(k-1)} \cdots A_{\varphi(0)}\right\|\right)^{\delta} \leq \\
& c_{2}\left(\beta_{1}+\beta_{2}\left\|A_{\varphi(k-1)}\right\|^{\delta}+\beta_{3}\left\|A_{\varphi(k-1)} A_{\varphi(k-2)}\right\|^{\delta}+\cdots\right. \\
& \left.+\beta_{k+1}\left\|A_{\varphi(k-1)} \cdots A_{\varphi(0)}\right\|^{\delta}\right) .
\end{aligned}
$$

For $\delta \leq 1$ we can still use the subadditivity to find $c_{2}=1$ and $\beta_{i}=1$ for all $i=1, \ldots, k+1$. For $\delta>1$ the convexity property would lead to a constant $c_{2}$ dependent of $k$ and hence to a divergent sum for $k \rightarrow \infty$. We, thus, make use of the result in Lemma 15 (see appendix) to fix $c_{2}=\zeta\left(\frac{\delta}{\delta-1}\right)^{\delta-1}$, with $\zeta(\cdot)$ the Euler-Riemann zeta function, and $\beta_{i}=i^{\delta}$. Hence, recalling that $d$ is deterministic, from (16) we have

$$
\begin{aligned}
\mathrm{E}\left\{\|x(k, d)\|^{\delta}\right\} & \leq c_{1} \mathrm{E}\left\{\left\|A_{\varphi(k-1)} \cdots A_{\varphi(0)}\right\|^{\delta}\right\}\left\|x_{0}\right\|^{\delta} \\
& +c_{1} c_{2} \bar{b}^{\delta}\|d\|_{\infty}^{\delta}\left(\beta_{1}+\beta_{2} \mathrm{E}\left\{\left\|A_{\varphi(k-1)}\right\|^{\delta}\right\}+\cdots\right.
\end{aligned}
$$

$$
\left.+\beta_{k+1} \mathrm{E}\left\{\left\|A_{\varphi(k-1)} \cdots A_{\varphi(0)}\right\|^{\delta}\right\}\right) .
$$

We must show that there exists a constant $c_{3} \in \mathbb{R}_{>0}$, independent of $k$ and $\beta_{i}$, such that $\beta_{1}+\beta_{2} \mathrm{E}\left\{\left\|A_{\varphi(k-1)}\right\|^{\delta}\right\}+$ $\cdots+\beta_{k+1} \mathrm{E}\left\{\left\|A_{\varphi(k-1)} \cdots A_{\varphi(0)}\right\|^{\delta}\right\} \leq c_{3}$. For the case $\delta \leq 1$ this is easily proved by means of the inequality (14). We have indeed (recall $\beta_{i}=1$ in this case): $1+\mathrm{E}\left\{\left\|A_{\varphi(k-1)}\right\|^{\delta}\right\}+$ $\cdots+\mathrm{E}\left\{\left\|A_{\varphi(k-1)} \cdots A_{\varphi(0)}\right\|^{\delta}\right\} \leq a \sum_{i=0}^{k} \mathrm{e}^{-b i}$. Hence we can fix $c_{3}=\frac{a}{1-\mathrm{e}^{-b}}$ by means of the geometric series. For $\delta>1$ we exploit again the inequality (14) to write $1+2^{\delta} \mathrm{E}\left\{\left\|A_{\varphi(k-1)}\right\|^{\delta}\right\}+3^{\delta} \mathrm{E}\left\{\left\|A_{\varphi(k-1)} A_{\varphi(k-2)}\right\|^{\delta}\right\}+\cdots+$ $(k+1)^{\delta} \mathrm{E}\left\{\left\|A_{\varphi(k-1)} \cdots A_{\varphi(0)}\right\|^{\delta}\right\} \leq a \sum_{i=0}^{k}(i+1)^{\delta} \mathrm{e}^{-b i}$. We finally have $a \sum_{i=0}^{k}(i+1)^{\delta} \mathrm{e}^{-b i}=a \mathrm{e}^{b} \sum_{j=1}^{k+1} j^{\delta} \mathrm{e}^{-b j} \leq c_{3}$ where $c_{3} \triangleq a \mathrm{e}^{b} \mathrm{Li}_{-\delta}\left(\mathrm{e}^{-b}\right)$ and $\operatorname{Li}_{s}(z)=\sum_{j=1}^{\infty} \frac{z^{j}}{j^{s}}$ is the polylogarithm (see Chapter 25 of [45]), which converges to a positive finite constant for all $0 \leq z<1$. Finally we have

$$
\mathrm{E}\left\{\|x(k, d)\|^{\delta}\right\} \leq a c_{1}\left\|x_{0}\right\|^{\delta} \mathrm{e}^{-b k}+c_{1} c_{2} c_{3} \bar{b}^{\delta}\|d\|_{\infty}^{\delta}
$$

where we can easily recognize the class $\mathcal{K}_{\infty}$ function $\gamma(s) \triangleq$ $c_{1} c_{2} c_{3} \bar{b}^{\delta} s^{\delta}$ for all $s \geq 0$. $\delta$-EISS follows.

We are now ready to provide sufficient conditions for the $\delta$ EISS (hence also for $\delta$-GAS, $\delta$-GES and $\delta$-ISS) of the system
(1). As a first step, we introduce a Lyapunov characterization for the $\delta$-EISS of system (1), which is in the same spirit of some classical results such as [46], [47].

Lemma 9. The system (1) driven by the stochastic process $\{\sigma(\cdot), \varphi(\cdot)\}$, whose distributions are described by the evolutions (3) and (4), is $\delta$-EISS if there exist functions $V_{i}: \mathbb{R}^{n} \rightarrow \mathbb{R}_{\geq 0}$, constants $\underline{\alpha}_{i}, \bar{\alpha}_{i}, \alpha_{i} \in \mathbb{R}_{>0}, i \in J_{\sigma}$, and a function $\chi \in \mathcal{K}_{\infty}$ such that for all $x \in \mathbb{R}^{n}$, all $d \in \mathbb{R}^{p}$ and all $k \in \mathbb{Z}_{>0}$,

i) $\quad \underline{\alpha}_{i}\|x\|^{\delta} \leq V_{i}(x) \leq \bar{\alpha}_{i}\|x\|^{\delta}$ for all $i \in J_{\sigma}$;

ii) $\quad \mathrm{E}\left\{V_{\sigma(k+1)}\left(A_{\varphi(k)} x+B(x, k) d(k)\right)-V_{\sigma(k)}(x)\right\} \leq$ $-\alpha_{i}\|x\|^{\delta}+\chi(\|d(k)\|)$.

Proof: Let us pick $\alpha_{1} \triangleq \min _{i \in J_{\sigma}} \underline{\alpha}_{i}, \alpha_{2} \triangleq \max _{i \in J_{\sigma}} \bar{\alpha}_{i}$ and $\alpha \triangleq \min _{i \in J_{\sigma}} \alpha_{i}$. Applying the expectation to both members of inequality ii) we have

$$
\begin{gathered}
\mathrm{E}\left\{\mathrm{E}\left\{V_{\sigma(k+1)}\left(A_{\varphi(k)} x(k)+B(x(k), k) d(k)\right)-V_{\sigma(k)}(x(k))\right\}\right\} \leq \\
-\alpha \mathrm{E}\left\{\|x(k)\|^{\delta}\right\}+\mathrm{E}\{\chi(\|d(k)\|)\}
\end{gathered}
$$

and thus

$$
\begin{array}{r}
\mathrm{E}\left\{V_{\sigma(k+1)}(x(k+1, d))\right\} \leq \\
\left(1-\frac{\alpha}{\alpha_{2}}\right) \mathrm{E}\left\{V_{\sigma(k)}(x(k, d))\right\}+\mathrm{E}\{\chi(\|d(k)\|)\} .
\end{array}
$$

By recursively solving the previous relation, we have

$$
\begin{aligned}
\mathrm{E}\left\{V_{\sigma(k)}(x(k, d))\right\} & \leq \rho^{k} \mathrm{E}\left\{V_{\sigma(0)}\left(x_{0}\right)\right\} \\
& +\sum_{j=0}^{k-1} \rho^{k-1-j} \mathrm{E}\{\chi(\|d(j)\|)\}
\end{aligned}
$$

with $\rho \triangleq\left(1-\frac{\alpha}{\alpha_{2}}\right)<1$. It is worth noting that $\rho \geq 0$. Indeed, by picking (18) with $d \equiv 0$, we get $\mathrm{E}\left\{V_{\sigma(k+1)}(x(k+1))\right\} \leq$ $\rho \mathrm{E}\left\{V_{\sigma(k)}(x(k))\right\}$. Since $\mathrm{E}\left\{V_{\sigma(k+1)}(x(k+1))\right\} \geq 0$ and $\mathrm{E}\left\{V_{\sigma(k)}(x(k))\right\} \geq 0$, it follows that $\rho \geq 0$. From (19) and recalling that $d$ is a deterministic input, we have

$$
\alpha_{1} \mathrm{E}\left\{\|x(k, d)\|^{\delta}\right\} \leq \alpha_{2} \rho^{k}\left\|x_{0}\right\|^{\delta}+\frac{1}{1-\rho} \chi\left(\|d\|_{\infty}\right) .
$$

Definition 6-2 is thus satisfied with

$$
\begin{aligned}
a & =\frac{\alpha_{2}}{\alpha_{1}}, \quad b=-\log \left(1-\frac{\alpha}{\alpha_{2}}\right) \\
\gamma(\cdot) & =\frac{\alpha_{2}}{\alpha_{1} \alpha} \chi(\cdot) .
\end{aligned}
$$

The next result provides an alternative Lyapunov condition for $\delta$-EISS. Contrarily to Lemma 9, it does not rely on the decrease of the Lyapunov function expectation, but rather involves the transition probabilities $p_{l i}$.

Lemma 10. The system (1) driven by the stochastic process $\{\sigma(\cdot), \varphi(\cdot)\}$, whose distributions are described by the evolutions (3) and (4), is $\delta$-EISS if there exist $M$ matrices $R_{l}=R_{l}^{T}>0$ and $\alpha \in \mathbb{R}_{>0}$ such that for all $x \in \mathbb{R}^{n}$ and for all $l \in J_{\sigma}$

$$
\sum_{h=1}^{N} \sum_{j=1}^{M} p_{l j} \ell_{l h}\left(x^{T} A_{h}^{T} R_{j} A_{h} x\right)^{\frac{\delta}{2}}-\left(x^{T} R_{l} x\right)^{\frac{\delta}{2}} \leq-\alpha\|x\|^{\delta},
$$


where we recall that $p_{l j} \triangleq \mathrm{P}\{\sigma(k+1)=j \mid \sigma(k)=l\}$ and $\ell_{l h} \triangleq \mathrm{P}\{\varphi(k)=h \mid \sigma(k)=l\}$.

Proof: In the light of Theorem 8 we can simply prove that the system is $\delta$-GES. Let us define the following candidate Lyapunov functions $V_{l}(x) \triangleq\left(x^{T} R_{l} x\right)^{\frac{\delta}{2}}, l \in J_{\sigma}$. They satisfy condition i) of Lemma 9 with $\alpha_{1}=\min _{j \in J_{\sigma}} \lambda_{\min }\left(R_{j}\right)$ and $\alpha_{2}=\max _{j \in J_{\sigma}} \lambda_{\max }\left(R_{j}\right)$. In order to prove the thesis, we need to verify condition ii) of the same lemma considering $d \equiv 0$. That is, for any $x \in \mathbb{R}^{n}$

$$
\begin{aligned}
\mathrm{E} & \left\{V_{\sigma(k+1)}\left(A_{\varphi(k)} x\right)-V_{\sigma(k)}(x)\right\} \\
& =\sum_{l=1}^{M} \mathrm{E}\left\{V_{\sigma(k+1)}\left(A_{\varphi(k)} x\right)-V_{\sigma(k)}(x) \mid \sigma(k)=l\right\} \mathrm{P}\{\sigma(k)= \\
& =\sum_{l=1}^{M} \mathrm{E}\left\{\left(x^{T} A_{\varphi(k)}^{T} R_{\sigma(k+1)} A_{\varphi(k)} x\right)^{\frac{\delta}{2}}\right. \\
& \left.-\left(x^{T} R_{\sigma(k)} x\right)^{\frac{\delta}{2}} \mid \sigma(k)=l\right\} \mathrm{P}\{\sigma(k)=l\} \\
& =\sum_{l=1}^{M}\left[\sum_{h=1}^{N} \sum_{j=1}^{M} \mathrm{P}\{\varphi(k)=h, \sigma(k+1)=j \mid \sigma(k)=l\}\right. \\
& \left.\times\left(x^{T} A_{h}^{T} R_{j} A_{h} x\right)^{\frac{\delta}{2}}-\left(x^{T} R_{l} x\right)^{\frac{\delta}{2}}\right] \mathrm{P}\{\sigma(k)=l\} \\
& =\sum_{l=1}^{M}\left[\sum_{h=1}^{N} \sum_{j=1}^{M} p_{l j} \ell_{l h}\left(x^{T} A_{h}^{T} R_{j} A_{h} x\right)^{\frac{\delta}{2}}-\left(x^{T} R_{l} x\right)^{\frac{\delta}{2}}\right] \\
& \times \mathrm{P}\{\sigma(k)=l\} \\
& \leq \sum_{l=1}^{M}\left(-\alpha\|x\|^{\delta}\right) \mathrm{P}\{\sigma(k)=l\}=-\alpha\|x\|^{\delta},
\end{aligned}
$$

where we used inequality (20), the fact that $\sum_{l=1}^{M} \mathrm{P}\{\sigma(k)=$ $l\}=1$ and also, recalling (3)-(4),

$$
\begin{aligned}
& \mathrm{P}\{\varphi(k)=h, \sigma(k+1)=j \mid \sigma(k)=l\} \\
& =\mathrm{P}\{\varphi(k)=h \mid \sigma(k+1)=j, \sigma(k)=l\} \\
& \times \mathrm{P}\{\sigma(k+1)=j \mid \sigma(k)=l\} \\
& =\mathrm{P}\{\varphi(k)=h \mid \sigma(k)=l\} \mathrm{P}\{\sigma(k+1)=j \mid \sigma(k)=l\} \\
& =\ell_{l h} p_{l j} .
\end{aligned}
$$

For the special case of 2-EISS $(\delta=2)$, the following LMI condition can be obtained using Lemma 10.

Theorem 11. The system (1) driven by the stochastic process $\{\sigma(\cdot), \varphi(\cdot)\}$, whose distributions are described by the evolutions (3) and (4), is 2-EISS if there exist $M$ matrices $R_{l}=R_{l}^{T}>0$ such that

$$
\sum_{h=1}^{N} \ell_{l h} A_{h}^{T} \tilde{R}_{l} A_{h}-R_{l}<0, \quad \forall l \in J_{\sigma}
$$

with

$$
\tilde{R}_{l} \triangleq \sum_{j=1}^{M} p_{l j} R_{j}, \quad \forall l \in J_{\sigma},
$$

where we recall that $p_{l j} \triangleq \mathrm{P}\{\sigma(k+1)=j \mid \sigma(k)=l\}$ and $\ell_{l h} \triangleq \mathrm{P}\{\varphi(k)=h \mid \sigma(k)=l\}$.

The proof is straightforward in view of Lemma 10. It is worth stressing that, if the matrix $L=\left(\ell_{l h}\right)_{\substack{l=1, \ldots, M \\ h=1, \ldots, N}}$ is the identity matrix, then the LMI conditions (21)- $(22)$ become the one of the second moment stability [25], [48], [49], [23].

\section{B. Almost Sure ISS}

The previous section shows, for the class of systems (1), the equivalence between $\delta$-stability properties (namely, $\delta$-GAS and $\delta$-GES) and robustness to exogenous inputs ( $\delta$-ISS and $\delta$ $l$ EISS), and provides natural Lyapunov tools to guarantee them. We now proceed in conducting a similar analysis for as-ISS. As a first step, we prove the following result.

Theorem 12. For the system (1) driven by the stochastic process $\{\sigma(\cdot), \varphi(\cdot)\}$, whose distributions are described by the evolutions (3) and (4), any property among $\delta$-GAS, $\delta$-GES, $\delta$-ISS and $\delta$-EISS implies as-ISS.

Proof: It is well known (see for instance [44], [22], [23]) that $\delta$-GAS $\Rightarrow$ as-GS, hence we can assume that

$$
\lim _{k \rightarrow \infty}\|x(k, 0)\|=0 \quad \text { almost surely (a.s.). }
$$

This property, together with (15), implies that, for any $\varepsilon \in$ $(0,1)$, there exists $Q=Q(\varepsilon) \in \mathbb{Z}_{>0}$ such that for every $q \geq Q$ and every initial distribution $\pi_{\sigma 0} \in S^{M-1}$,

$$
\left\|A_{\varphi(q-1)} \cdots A_{\varphi(0)}\right\| \leq \varepsilon \quad \text { a.s. }
$$

The previous inequality is satisfied by almost any sequence $\varphi(0), \ldots, \varphi(q-1)$ generated by any initial distribution $\pi_{\sigma 0} \in$ $S^{M-1}$. Due to the arbitrariness of the initial distribution and the time-homogeneity property of the composite process $\{\sigma(\cdot), \varphi(\cdot)\}$, the same property holds for any sequence starting at any instant $s \in \mathbb{Z}_{>0}$. Or, equivalently, almost all sequences of length $q \geq Q$ of product of dynamic matrices satisfy such inequality. Hence, we have

$$
\left\|\prod_{j=0}^{q-1} A_{i_{j}}\right\| \leq \varepsilon \quad \text { a.s. } \quad \forall i_{j} \in J_{\varphi} .
$$

Moreover, there exists $c>1$ such that, for any $h \in \mathbb{Z}_{>0}$ with $h<Q$, we have

$$
\left\|\prod_{j=0}^{h-1} A_{i_{j}}\right\| \leq c \quad \text { a.s. } \quad \forall i_{j} \in J_{\varphi} .
$$

Using again the time-homogeneity property of $\{\sigma(\cdot), \varphi(\cdot)\}$, we can write for any $k=r Q+h, h, r \in \mathbb{Z}_{>0}, h<Q$

$$
\left\|A_{\varphi(k-1)} \cdots A_{\varphi(0)}\right\| \leq c \varepsilon^{r}=a \mathrm{e}^{-b k},
$$

with $a \triangleq c \varepsilon^{-\frac{h}{Q}}$ and $b \triangleq-\frac{1}{Q} \log \varepsilon$. Proceeding in a similar way as for the proof of Theorem 8 , we obtain the inequality $\|x(k, d)\| \leq \beta\left(k,\left\|x_{0}\right\|\right)+\gamma\left(\|d\|_{\infty}\right)$ almost surely, with the class $\mathcal{K} \mathcal{L}$ function $\beta(k, s) \triangleq a \mathrm{e}^{-b k} s$ and the class $\mathcal{K}_{\infty}$ function $\gamma(s) \triangleq \frac{a \bar{b}}{1-\mathrm{e}^{-b}} s$. 
Exploiting the previous theorem and Lemma 10 and proceeding as in Theorem 2.1 in [50], we can provide sufficient conditions for the as-ISS of our system. In case $L$ is the identity matrix, such conditions turn to those provided in [50] for as-GS.

Corollary 13. The system (1) driven by the stochastic process $\{\sigma(\cdot), \varphi(\cdot)\}$, whose distributions are described by the evolutions (3) and (4), is as-ISS if there exist $M$ matrices $R_{l}=R_{l}^{T}>0$ such that one of the following conditions is verified

$$
\begin{aligned}
& \text { - } \max _{\|x\|=1} \prod_{h=1}^{N} \prod_{j=1}^{M}\left(\frac{x^{T} A_{h}^{T} R_{j} A_{h} x}{x^{T} R_{l} x}\right)^{p_{l j} \ell_{l h}}<1, \quad \forall l \in J_{\sigma} \\
& \text { - } \quad \prod_{h=1}^{N} \prod_{j=1}^{M} \lambda_{\max }\left(A_{h}^{T} R_{j} A_{h} R_{l}^{-1}\right)^{p_{l j} \ell_{l h}}<1, \quad \forall l \in J_{\sigma} .
\end{aligned}
$$

As remarked in Section III, if $\{\sigma(\cdot)\}$ is a FSHIA-MC, thus an ergodic process with a unique ergodic class and a unique i.p.d. $\bar{\pi}_{\sigma}$, the process $\{\varphi(\cdot)\}$ inherits the same ergodic property and it has a unique i.p.d. $\bar{\pi}_{\varphi}=\bar{\pi}_{\sigma} L$. Therefore, for ergodic processes we have the following condition (see also [22], [50]).

Corollary 14. The system (1) driven by the stochastic ergodic process $\{\sigma(\cdot), \varphi(\cdot)\}$, whose distributions are described by the evolutions (3) and (4) and whose i.p.d. is given by $\left(\bar{\pi}_{\sigma}, \bar{\pi}_{\varphi}\right)$ with $\bar{\pi}_{\varphi} \triangleq\left[\bar{\pi}_{\varphi_{1}}, \ldots, \bar{\pi}_{\varphi_{M}}\right]=\bar{\pi}_{\sigma} L$, is as-ISS if

$$
\prod_{j=1}^{M}\left\|A_{j}\right\|^{\bar{\pi}_{\varphi_{j}}}<1 .
$$

\section{CASE STUDY}

In this section we illustrate the robustness properties guaranteed by the 2-EISS conditions in Theorem 11 on a tracking problem for the benchmark mechanical system in Figure 2. We remark that the TORA is a planar system where the rotating arm is implanted above the oscillating mass. Hence, the gravity force is orthogonal to the plan.

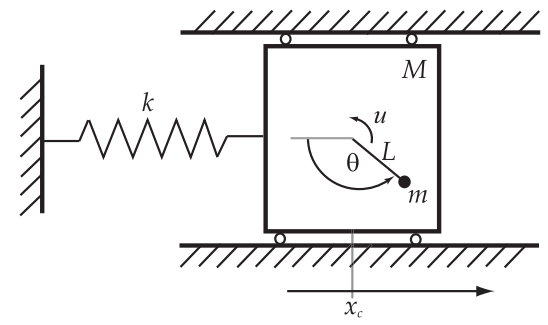

Fig. 2. Model of a Translational Oscillator/Rotational Actuator (TORA) system ([51])

\section{A. Plant and control description}

The Translational Oscillator/Rotational Actuator (TORA) system in Figure 2 (see [51] for further details) can be described as follows

$$
\begin{aligned}
{\left[\begin{array}{c}
\ddot{\theta} \\
\ddot{x}_{c}
\end{array}\right] } & =\frac{1}{\Delta(\theta)}\left[\begin{array}{cc}
m+M & -m L \cos \theta \\
-m L \cos \theta & I+m L^{2}
\end{array}\right]\left[\begin{array}{c}
u+d \\
m L \dot{\theta}^{2} \sin \theta-\kappa x
\end{array}\right] \\
y & =\theta+n
\end{aligned}
$$

where $\Delta(\theta)=\left(I+m L^{2}\right)(m+M)-m^{2} L^{2} \cos ^{2} \theta>0, M$ is the mass of the translational oscillator, $m$ and $I$ are the mass and the inertia of the rotational actuator located at a distance $L$ from the center of rotation, $\kappa$ is the stiffness of the spring, $\theta$ is the angle of the actuator, $x_{c}$ is the horizontal displacement of the oscillator, $u$ is the control torque, $d$ is the torque disturbance and $n$ is the measurement error. The previous parameters are assumed to take the following values throughout this section: $m=1 \mathrm{~kg}, M=5 \mathrm{~kg}, L=0.1 \mathrm{~m}$, $I=0.01 \mathrm{~kg} \mathrm{~m}^{2}$ and $\kappa=20 \mathrm{~N} / \mathrm{m}$. After linearization about the origin and sampled-time discretization with sampling time $T=0.1 \mathrm{~s}$, we obtain the following transfer function from $u$ to $\theta$

$$
G(z)=\frac{0.27266(z+1)\left(z^{2}-1.967 z+1\right)}{(z-1)^{2}\left(z^{2}-1.964 z+1\right)} .
$$

We assume measurements to be acquired at the beginning of each period and control inputs to be released at the end, thus the controller is not affected by jitter but experiences a constant unit delay. In order to account for the unit delay, controllers are designed for the transfer function $G(z) \frac{1}{z}$ instead of $G(z)$.

We assume here that the control task is designed according to the structure illustrated in Example 1. In particular, the controller $\Gamma_{1}(z)=K_{1}(z)=\frac{3.04(z-0.97)}{z+0.9}$ is designed to ensure only stability requirement, while the second controller $\Gamma_{2}(z)=K_{1}(z)+K_{2}(z)$ with $K_{2}(z)=\frac{-0.021(z-2)}{z-0.76}$ to enhance performance in terms of rise time and settling time (see Figure 3 for a graphical comparison). The prefilters $\Phi_{1}(z)=F_{1}(z)=0.0480$ and $\Phi_{2}(z)=F_{1}(z) F_{2}(z)=0.1351$ are used to adapt the steady-state gain and ensure static requirements.

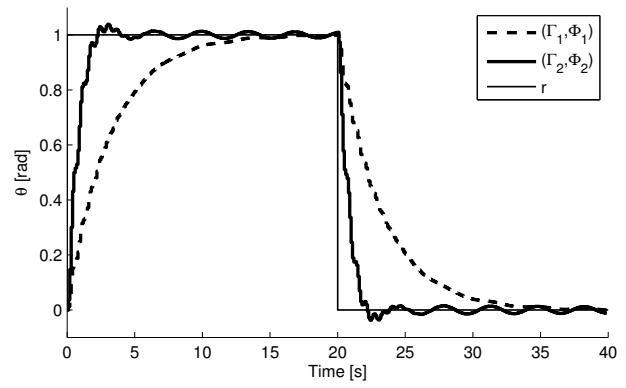

Fig. 3. Outputs of the TORA system in closed loop with $\left(\Gamma_{1}, \Phi_{1}\right)$ and $\left(\Gamma_{2}, \Phi_{2}\right)$ for a square wave reference $r$ of period $40 \mathrm{~s}$, duty cycle $50 \%$ and amplitude $1 \mathrm{rad}$.

According to the anytime paradigm, the task implementing the controllers has to minimize the execution time, thus the computations, while still preserving numerical robustness. To this aim, in [12, Sec. VII] the controllers were implemented in Jordan state-space realization. For more details on the structure of the two closed loop matrices of the linearized system with the two controllers see [12, Sec. VII].

\section{B. Stochastic description of the computation platform}

In this case study we have two controllers $\Gamma_{1}$ and $\Gamma_{2}$, hence $J_{\varphi} \triangleq\{1,2\}$. We also assume that the available computation time can take four different values, hence $J_{\sigma} \triangleq\{1, \ldots, 4\}$. We 
assume the FSH-MC $\{\sigma(\cdot)\}$ to be described by the following transition probability matrix

$$
P=\left[\begin{array}{cccc}
0.5 & 0.1 & 0.4 & 0 \\
0.2 & 0.1 & 0.5 & 0.2 \\
0.2 & 0.2 & 0.4 & 0.2 \\
0.1 & 0.2 & 0.3 & 0.4
\end{array}\right]
$$

and the following initial probability distribution $\pi_{\sigma 0}=$ $\frac{1}{4}[1,1,1,1]$ (see [13] for more details on how to construct the transition probability matrix). The stationary probability distributions of the execution times $T^{1}$ and $T^{2}$ are given by the row vectors $\pi_{T^{1}}=[1,0,0,0]$ and $\pi_{T^{2}}=[0,0.33,0.5,0.17]$ respectively. Therefore, the matrix $L$ in (4) is

$$
L=\left[\begin{array}{cc}
1 & 0 \\
0.67 & 0.33 \\
0.17 & 0.83 \\
0 & 1
\end{array}\right]
$$

The controller actually executed at each time step $k$ is the largest possible one within the available computation time. That is, if the available computation time $\sigma(k)$ at step $k$ is such that $\sigma(k) \geq T^{2}(k)$, then $\varphi(k)=2$, otherwise $\varphi(k)=1$. We assume here that $\sigma(k) \geq T^{1}(k)$ for every $k$.

\section{Simulation results}

The closed-loop SJLS with the computation platform described in the previous sections satisfies the LMI conditions of Theorem 11, thus it turns out to be 2-EISS. For simulation purposes we have considered a tracking problem with a square wave reference of period $40 \mathrm{~s}$, duty cycle $50 \%$ and amplitude $1 \mathrm{rad}$. We assume also that the input disturbance $d$ and the measurement error $n$ affecting the SJLS (see the scheme in Figure 1) are bounded as $|d| \leq 0.05 \mathrm{Nm}$ and $|n| \leq 0.05 \mathrm{rad}$. Figure 4 shows a simulation run for the TORA system in closed loop with $\left(\Gamma_{1}, \Phi_{1}\right)$, with $\left(\Gamma_{2}, \Phi_{2}\right)$ and with the anytime control (SJLS). The vertical black segments of the graph on the bottom represent the instants where $\left(\Gamma_{1}, \Phi_{1}\right)$ is executed, that is the steps $k$ where $\varphi(k)=1$. The empty spaces among those segments represent, instead, the steps $k$ where $\varphi(k)=2$. Simulations show a bounded response in presence of the bounded disturbances, which is typical of an ISS behavior.

Figure 5 illustrates the first 4 seconds of temporal evolution of a sample path for the processes $T^{1}(\cdot), T^{2}(\cdot)$ and $\sigma(\cdot)$. The available $(\sigma(\cdot))$ and required $\left(T^{1}(\cdot), T^{2}(\cdot)\right)$ computation time range in the discrete set $J_{\sigma}=\{1, \ldots, 4\}$. Whenever $\sigma(k) \geq T^{2}(k)$, it holds that $\varphi(k)=2$, otherwise $\varphi(k)=1$. The process $\varphi(\cdot)$ is represented at the bottom of the figure as a bichromatic thick line: dark gray segments accounts for the activation periods of $\left(\Gamma_{2}, \Phi_{2}\right)$, light gray segments indicate those of $\left(\Gamma_{1}, \Phi_{1}\right)$.

This example shows the applicability of our result and its ability to formally guarantee robustness properties.

\section{CONCLUSIONS}

In this paper we have introduced a model of SJLS encompassing as special cases switching systems driven by MCs and i.i.d. processes. Such a model proves very useful in

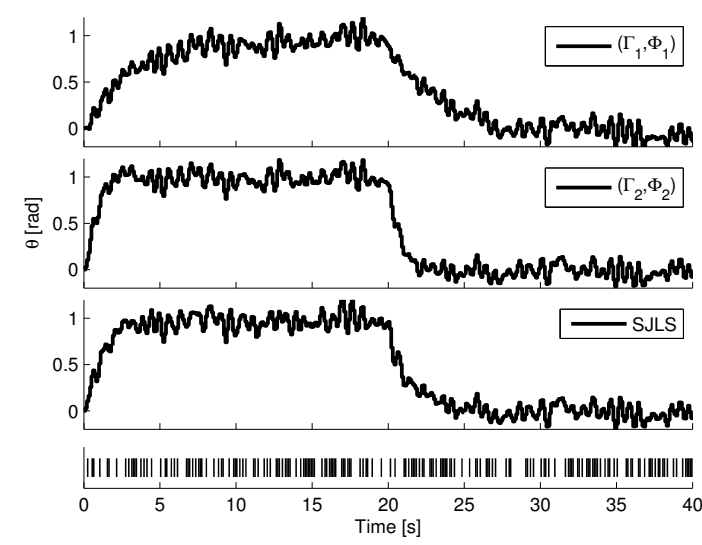

Fig. 4. Outputs of the TORA system in closed loop with $\left(\Gamma_{1}, \Phi_{1}\right),\left(\Gamma_{2}, \Phi_{2}\right)$ and with the anytime control for a square wave reference of period $40 \mathrm{~s}$, duty cycle $50 \%$ and amplitude $1 \mathrm{rad}$. The system is affected by bounded input disturbance $d$ and measurement error $n$ with $|d| \leq 0.05 \mathrm{Nm}$ and $|n| \leq$ $0.05 \mathrm{rad}$. The bottom graph portraits the activation instants of $\left(\Gamma_{1}, \Phi_{1}\right)$.

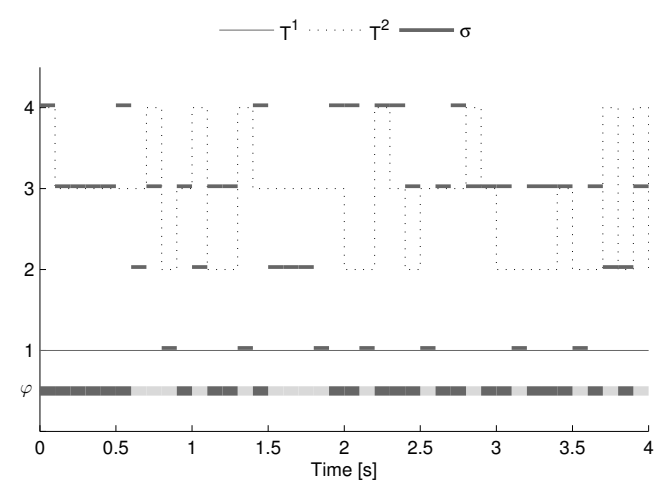

Fig. 5. First 4 seconds of temporal evolution of a sample path for the processes $T^{1}(\cdot), T^{2}(\cdot)$ and $\sigma(\cdot)$. Whenever $\sigma(k) \geq T^{2}(k)$, it holds that $\varphi(k)=2$, otherwise $\varphi(k)=1$. The bottom graph represents the process $\varphi(\cdot)$ : dark gray segments accounts for the activation periods of $\left(\Gamma_{2}, \Phi_{2}\right)$, light gray segments indicate those of $\left(\Gamma_{1}, \Phi_{1}\right)$.

describing the varying behavior of shared resource availability, which characterizes many cyber-physical systems. We have also presented stochastic ISS definitions which smoothly connect deterministic ISS results and classical stochastic stability definitions for SJLSs. These definitions come with easily testable sufficient conditions for the aforementioned more general SJLS. In the future we aim at further extending the stochastic model to catch the complex behaviors arising when the interaction of different SJLSs on the shared resource induces a correlation among the stochastic properties of the SJLSs. Quantitative performance metrics will be investigated as well.

\section{APPENDIX}

Lemma 15. Given any $k \in \mathbb{Z}_{\geq 0}$, any $z_{i} \in \mathbb{R}_{\geq 0}, i=1, \ldots, k$, and any $\delta>1$ the following inequality holds

$$
\left(\sum_{i=1}^{k} z_{i}\right)^{\delta} \leq \zeta\left(\frac{\delta}{\delta-1}\right)^{\delta-1} \sum_{i=1}^{k} i^{\delta} z_{i}^{\delta}
$$


where $\zeta(\cdot)$ is the Euler-Riemann zeta function. Notice that, since $\delta>1, \zeta\left(\frac{\delta}{\delta-1}\right)$ is a positive finite constant independent of $z_{i}$ and $k$.

We start by showing that, given any $\delta>1$ and any $\beta=$ $\left(\beta_{1}, \ldots, \beta_{k}\right)^{T} \in \mathbb{R}_{>0}^{k}$, there exists a constant $c(\delta, \beta, k)>0$ such that, for any $w=\left(w_{1}, \ldots, w_{k}\right)^{T} \in \mathbb{R}_{\geq 0}^{k}$ satisfying $\sum_{i=1}^{k} w_{i}=1$,

$$
\sum_{i=1}^{k} \beta_{i} w_{i}^{\delta} \geq \frac{1}{c(\delta, \beta, k)} .
$$

To that aim, we compute the following minimum:

$$
\min \left\{\sum_{i=1}^{k} \beta_{i} w_{i}^{\delta} \mid \sum_{i=1}^{k} w_{i}=1, w_{i} \geq 0\right\} .
$$

By means of the Lagrange multipliers we define

$$
\Lambda(w, \lambda) \triangleq \sum_{i=1}^{k} \beta_{i} w_{i}^{\delta}+\lambda\left(\sum_{i=1}^{k} w_{i}-1\right) .
$$

Solving $\nabla \Lambda=0$ leads to the conditions

$$
\begin{aligned}
\beta_{i} \delta w_{i}^{\delta-1}+\lambda & =0 \quad \forall i=1, \ldots, k \\
\sum_{i=1}^{k} w_{i} & =1 .
\end{aligned}
$$

From (27) we have that, for any $i, j \in\{1, \ldots, k\}, \beta_{i} w_{i}^{\delta-1}=$ $\beta_{j} w_{j}^{\delta-1}$, and thus

$$
w_{i}=\left(\frac{\beta_{j}}{\beta_{i}}\right)^{\frac{1}{\delta-1}} w_{j}
$$

Replacing (29) in (28), we get that the minimum (26) is reached for $w=w^{\star}$, where

$$
w_{j}^{\star}=\frac{1}{\beta_{j}^{\frac{1}{\delta-1}} \sum_{i=1}^{k} \frac{1}{\beta_{i}^{\frac{1}{\delta-1}}}}, \quad \forall j=1, \ldots, k .
$$

We conclude that the sought constant $c$ in (25) can be picked as

$$
\begin{aligned}
c(\delta, \beta, k) & =\frac{1}{\sum_{i=1}^{k} \beta_{i}\left(w_{i}^{\star}\right)^{\delta}} \\
& =\frac{\left(\sum_{i=1}^{k} \frac{1}{\beta_{i}^{\frac{1}{\delta-1}}}\right)^{\delta}}{\sum_{j=1}^{k} \frac{1}{\beta_{j}^{\frac{1}{\delta-1}}}} \\
& =\left(\sum_{i=1}^{k} \frac{1}{\beta_{i}^{\frac{1}{\delta-1}}}\right)^{\delta-1} .
\end{aligned}
$$

Now, notice that if $z_{i}=0$ for all $i \in\{1, \ldots, k\}$, then the inequality (24) is trivially satisfied. If, on the other hand, $z_{i} \neq$ 0 for some $i$ then let

$$
w_{i}=\frac{z_{i}}{\sum_{i=1}^{k} z_{i}} \geq 0, \quad \forall i=1, \ldots, k,
$$

which indeed satisfy $\sum_{i=1}^{k} w_{i}=1$. Then, by considering the bound (25) with $\beta_{i}=i^{\delta}$, we obtain that

$$
\sum_{i=1}^{k} i^{\delta} z_{i}^{\delta} \geq \frac{\left(\sum_{i=1}^{k} z_{i}\right)^{\delta}}{c(\delta, \ell, k)}
$$

where $\ell(\delta, k) \triangleq\left(1^{\delta}, 2^{\delta}, \ldots, k^{\delta}\right)^{T}$. Furthermore, notice that

$$
\begin{aligned}
c(\delta, \ell, k) & =\left(\sum_{i=1}^{k} \frac{1}{i^{\frac{\delta}{\delta-1}}}\right)^{\delta-1} \\
& \leq\left(\sum_{i=1}^{\infty} \frac{1}{i^{\frac{\delta}{\delta-1}}}\right)^{\delta-1} \\
& \leq \zeta\left(\frac{\delta}{\delta-1}\right)^{\delta-1} .
\end{aligned}
$$

We conclude, in view of (31), that

$$
\left(\sum_{i=1}^{k} z_{i}\right)^{\delta} \leq c(\delta, \ell, k) \sum_{i=1}^{k} i^{\delta} z_{i}^{\delta} \leq \zeta\left(\frac{\delta}{\delta-1}\right)^{\delta-1} \sum_{i=1}^{k} i^{\delta} z_{i}^{\delta} .
$$

\section{ACKNOWLEDGMENT}

The authors would like to thank Paolo Mason for his important help in devising Lemma (15).

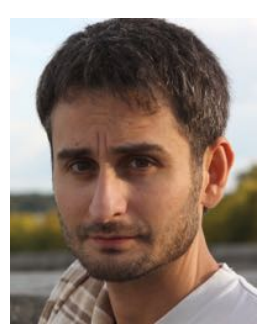

Luca Greco received the M.S. degree in Computer Engineering and the Ph.D. degree in Automation, Robotics and Bioengineering from the University of Pisa, Pisa, Italy, in 2001 and 2005, respectively. From 2005 to 2007, he was a Post-Doc fellow at the Interdepartmental Research Center "E. Piaggio", University of Pisa. From 2007 to 2009, he was a Post-Doc fellow at the DIIMA, University of Salerno, Salerno, Italy and from 2009 to 2012 he was Post-Doc fellow at L2S - Supélec, Gif-sur-Yvette, France. Since September 2012 he has been serving as an Associate Professor at L2S - Univ. Paris Sud 11, Orsay, France. His research interests include stability analysis and stabilization of hybrid and switching systems, embedded system control and networked control systems.

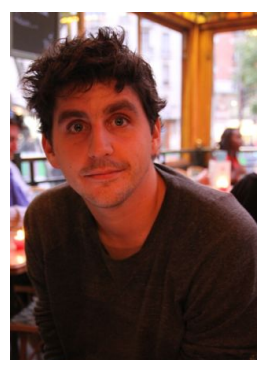

Antoine Chaillet was born in Douai (FR) in 1979. In 2002, he received his B.Sc. degree from ESIEE Amiens (FR), and his M.Sc. degree in Control Engineering from Univ. Paris Sud (FR) in 2003. He was an undergraduate scholar visitor to the University of Twente (NL), and a graduate scholar visitor to the INRIA Sophia Antipolis (FR). In July 2006, he received his Ph.D. degree cum laude in Control Theory from Univ. Paris Sud-L2S (FR). In 2004, A. Chaillet was recipient of a Marie-Curie Scholarship to visit Università degli Studi di Firenze (IT). In 2006-2007, he served as a post-doc fellow at Centro di Ricerca Piaggio, Pisa (IT). Since Sept. 2007, he has been serving as an associate professor at L2S-Univ. Paris Sud-CentraleSupélec (FR). His research interests include control theory for neuroscience applications, stability analysis and stabilization of nonlinear systems, control of mechanical systems, and network controlled systems. He is the author of 60 peer-reviewed publications on these topics. 


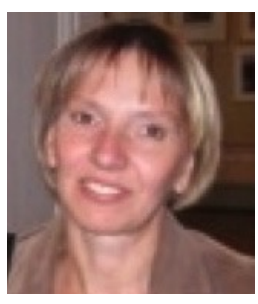

Elena Panteley (M.Sc. '86, Ph.D. '97) is an associate researcher of the French National Centre of Scientific Research (CNRS), Laboratoire de Signaux et Systèmes, France. She received her M.Sc. and $\mathrm{Ph} . \mathrm{D}$. degrees in applied mathematics from the State University of St. Petersburg, St. Petersburg, Russia. From 1986 to 1998, she held a research position with the Institute for Problem of Mechanical Engineering, Russian Academy of Science, St. Petersburg. Her research interests include stability and control of nonlinear dynamical systems, network systems with applications to electro-mechanical and neuronal systems.

\section{REFERENCES}

[1] J. A. Cook, I. V. Kolmanovsky, D. McNamara, E. C. Nelson, and K. V. Prasad, "Control, computing and communications: Technologies for the twenty-first century model T," Proc. of IEEE, vol. 95, no. 2, pp. 334 355, Feb. 2007.

[2] J. R. Moyne and D. M. Tilbury, "The emergence of industrial control networks for manufacturing control, diagnostics, and safety data," Proc. of IEEE, vol. 95, no. 1, pp. 29-47, 2007.

[3] S. Manolache, P. Eles, and Z. Peng, "Memory and time-efficient schedulability analysis of task sets with stochastic execution time," in Proc. 13th Euromicro Conf. on Real-Time Systems, 2001, pp. 19-26.

[4] G. Bernat, A. Colin, and S. M. Petters, "WCET analysis of probabilistic hard real-time systems," in 23rd IEEE Real-Time Systems Symposium (RTSS 2002), 2002, pp. 279-288.

[5] — " "pWCET: A tool for probabilistic worst-case execution time analysis of real-time systems," University of York, Department of Computer Science, Tech. Rep. YCS353, 2003.

[6] J. L. Diaz, D. F. Garcia, K. Kim, C.-G. Lee, L. L. Bello, J. M. Lopez, S. L. Min, and O. Mirabella, "Stochastic analysis of periodic real-time systems," in 23rd IEEE Real-Time Systems Symposium (RTSS 2002), 2002, pp. 289-300.

[7] D. Liu, X. S. Hu, M. D. Lemmon, and Q. Ling, "Firm real-time system scheduling based on a novel QoS constraint," IEEE Trans. on Computers, vol. 55, no. 3, pp. 320-333, March 2006.

[8] K. Kim, J. L. Diaz, L. L. Bello, J. M. Lopez, C.-G. Lee, and S. L. Min, "An exact stochastic analysis of priority-driven periodic real-time systems and its approximations," IEEE Trans. on Computers, vol. 54, no. 11, pp. 1460-1466, Nov. 2005.

[9] Q. Ling and M. D. Lemmon, "Soft real-time scheduling of networked control systems with dropouts governed by a Markov chain," in Proc. American Control Conf., vol. 6, 4-6 June 2003, pp. 4845-4850.

[10] T. Chantem, X. S. Hu, and M. Lemmon, "Generalized elastic scheduling for real-time tasks," IEEE Trans. on Computers, vol. 58, no. 4, pp. 480495, april 2009

[11] J. Nilsson and B. Bernhardsson, "Analysis of real-time control systems with time delays," in Proc. IEEE Int. Conf. on Decision and Control, 1996, pp. 3173-3178.

[12] L. Greco, D. Fontanelli, and A. Bicchi, "Design and stability analysis for anytime control via stochastic scheduling," IEEE Trans. on Automat. Contr., vol. 56, no. 3, pp. 571-585, 2010.

[13] A. Quagli, D. Fontanelli, L. Greco, L. Palopoli, and A. Bicchi, "Design of embedded controllers based on anytime computing," IEEE Trans. Industrial Infor, vol. 6, no. 4, pp. 492-502, Nov. 2010.

[14] D. Fontanelli, L. Greco, and L. Palopoli, "Adaptive reservations for feedback control," in Proc. 49th IEEE Int. Conf. on Decision and Control, Atlanta, GA, USA, December 2010, pp. 4236-4243.

[15] D. Fontanelli, L. Palopoli, and L. Greco, "Deterministic and stochastic QoS provision for real-time control systems," in 17th IEEE RTAS, Chicago, IL, USA, April 2011, pp. 103-112.

[16] D. E. Quevedo and V. Gupta, "Sequence-based anytime control," IEEE Trans. on Automat. Contr., vol. 58, no. 2, pp. 377-390, 2013.

[17] F. Kozin, "A survey of stability of stochastic systems," Automatica, vol. 5, no. 1, pp. 95-112, 1969.

[18] H. J. Kushner, Stochastic stability and control, ser. Mathematics in science and engineering. Academic Press, 1967.

[19] C. Kubrusly and O. L. V. Costa, "Mean square stability conditions for discrete stochastic bilinear systems," IEEE Trans. on Automat. Contr., vol. 30, no. 11, pp. 1082-1087, Nov. 1985.

[20] D. Chatterjee and D. Liberzon, "Stabilizing randomly switched systems," SIAM J. Contr. Optim., vol. 49, no. 5, pp. 2008-2031, 2011.

[21] Y. Fang, "Stability analysis of linear control systems with uncertain parameters," Ph.D. dissertation, Case Western Reserve University, 1994.
[22] Y. Fang, K. A. Loparo, and X. Feng, "Almost sure and $\delta$-moment stability of jump linear systems," Int. J. Contr., vol. 59, no. 5, pp. 12811307, 1994.

[23] — , "Stability of discrete time jump linear systems," J. Math. Sys. Estim. Contr, vol. 5, no. 3, pp. 275-321, 1995.

[24] Y. Fang and K. A. Loparo, "Stochastic stability of jump linear systems," IEEE Trans. on Automat. Contr., vol. 47, no. 7, pp. 1204-1208, 2002.

[25] T. Morozan, "Optimal stationary control for dynamic systems with Markov perturbations," Stoch. Anal. Appl., vol. 3, no. 1, pp. 299-325, 1983.

[26] X. Feng, K. A. Loparo, Y. Ji, and H. J. Chizeck, "Stochastic stability properties of jump linear systems," IEEE Trans. on Automat. Contr., vol. 37, no. 1, pp. 38-53, 1992.

[27] Y. Fang and K. A. Loparo, "Stabilization of continuous-time jump linear systems," IEEE Trans. on Automat. Contr., vol. 47, no. 10, pp. 15901603, 2002.

[28] E. D. Sontag, "Smooth stabilization implies coprime factorization," IEEE Trans. on Automat. Contr., vol. 34, no. 4, pp. 435-443, apr 1989.

[29] J. Tsinias, "Stochastic input-to-state stability and applications to global feedback stabilization," Int. J. Contr., vol. 71, no. 5, pp. 907-930, Jan. 1998.

[30] - "The concept of "exponential input to state stability" for stochastic systems and applications to feedback stabilization," Systems \& Control Letters, vol. 36, pp. 221-229, 1999.

[31] J. Spiliotis and J. Tsinias, "Notions of exponential robust stochastic stability, ISS and their Lyapunov characterizations," Int. J. Robust and Nonlin. Contr., vol. 13, no. 2, pp. 173-187, 2003.

[32] P. Zhao, W. Feng, and Y. Kang, "Stochastic input-to-state stability of switched stochastic nonlinear systems," Automatica, vol. 48, no. 10, pp. 2569-2576, Oct. 2012.

[33] D. Chatterjee and D. Liberzon, "Towards ISS disturbance attenuation for randomly switched systems," in Proc. IEEE Int. Conf. on Decision and Control, New Orleans, LA, USA, 2007, pp. 5612-5617.

[34] M. Tanelli, B. Picasso, P. Bolzern, and P. Colaneri, "Almost sure stabilization of uncertain continuous-time Markov jump linear systems," IEEE Trans. on Automat. Contr., vol. 55, no. 1, pp. 195-201, 2010.

[35] O. L. V. Costa, M. D. Fragoso, and R. P. Marques, Discrete-time Markov jump linear systems. Springer \& Verlag, 2005.

[36] P. Billingsley, Probability and Measure, 3rd ed. John Wiley \& Sons, 1995.

[37] R. J. Elliott, L. Aggoun, and J. B. Moore, Hidden Markov Models: Estimation and Control, ser. Applications of mathematics. SpringerVerlag, 1995.

[38] L. Rabiner, "A tutorial on hidden markov models and selected applications in speech recognition," Proceedings of the IEEE, vol. 77, no. 2, pp. 257-286, 1989.

[39] W. Zucchini and I. MacDonald, Hidden Markov Models for Time Series: An Introduction Using $R$, ser. Chapman \& Hall/CRC Monographs on Statistics \& Applied Probability. Taylor \& Francis, 2009.

[40] P. Dymarski, Ed., Hidden Markov Models, Theory and Applications. InTech, 2011.

[41] C. D. Meyer, Matrix Analysis and Applied Linear Algebra. Philadelphia, PA, USA: SIAM, 2000.

[42] P. Bolzern, P. Colaneri, and G. De Nicolao, "Markov jump linear systems with switching transition rates: Mean square stability with dwell-time," Automatica, vol. 46, no. 6, pp. 1081-1088, Jun. 2010.

[43] P. S. Rossi, G. Romano, F. Palmieri, and G. Iannello, "Joint end-toend loss-delay hidden markov model for periodic udp traffic over the internet," IEEE Transactions on Signal Processing, vol. 54, no. 2, pp. 530-541, Feb. 2006.

[44] F. Kozin, "On relations between moment properties and almost sure Lyapunov stability for linear stochastic systems," J. Math. Anal. Applicat., vol. 10, pp. 324-353, 1965.

[45] F. W. J. Olver, D. W. Lozier, R. F. Boisvert, and C. W. Clark, Eds., NIST Handbook of Mathematical Functions. Cambridge University Press, 2010.

[46] H. J. Kushner, "On the stability of stochastic dynamical systems," Proc. Nat. Acad. of Sciences USA, vol. 53, no. 1, pp. 8-12, 1965.

[47] F. J. Beutler, "On two discrete-time system stability concepts and supermartingales," J. Math. Anal. Applicat., vol. 44, no. 2, pp. 464-471, 1973.

[48] Y. Ji and H. J. Chizeck, "Jump linear quadratic gaussian control: steadystate solution and testable conditions," Control Theory Adv. Technol., vol. 6, no. 3, pp. 289-318, 1990.

[49] O. L. V. Costa and M. D. Fragoso, "Stability results for discrete-time linear systems with Markovian jumping parameters," J. Math. Anal. Applicat., vol. 179, pp. 154-178, 1993. 
[50] Y. Fang, "A new general sufficient condition for almost sure stability of jump linear systems," IEEE Trans. on Automat. Contr., vol. 42, no. 3 , pp. 378-382, 1997.

[51] R. T. Bupp, D. S. Bernstein, and V. T. Coppola, "A benchmark problem for nonlinear control design," Int. J. Robust and Nonlin. Contr., vol. 8, no. 4-5, pp. 307-310, 1998. 